\title{
Family Seiberg-Witten invariants and wall crossing formulas
}

\author{
Tian-Jun Li And Ai-Ko LiU
}

\section{Introduction.}

The Seiberg-Witten theory was initially introduced by Seiberg and Witten ([SW], [W]) and has led to many exciting results of smooth four manifolds. When $b^{+}$of a four manifold is larger than one, the original Seiberg-Witten invariants constitute a map from the set of $\operatorname{Spin}^{c}$ structures on the four manifold to the integers. And when the first betti number of the four manifold $M$ is positive, there is an extension of SW to a map (still denoted by $\mathrm{SW}$ ) to $\Lambda^{*} H^{1}(M ; \mathbf{Z})=\mathbf{Z} \oplus H^{1} \oplus \cdots \oplus \Lambda^{b_{1}} H^{1}$. In the case when $b^{+}$is equal to one, the map SW depends also on a choice of chamber. On a symplectic manifold, as observed in [T1], there is a canonical idenification of the set of Spin $^{c}$ structures with $H^{2}(M ; \mathbf{Z})$ and the symplectic form picks up a unique chamber. Thus, on a symplectic manifold, $\mathrm{SW}$ can be viewed as a map from $H^{2}(M ; \mathbf{Z})$ to $\Lambda^{*} H^{1}(M ; \mathbf{Z})$.

In a series of remarkable works [T1]-[T5], Taubes develops the SeibergWitten theory on symplectic manifolds. In [T2], he defines the GromovTaubes invariants of symplectic 4-manifolds counting embedded, (but not necessarily connected) pseudo-holomorphic curves. (It is recently proved in [IP] that the Gromov-Taubes invariants can also be constructed from the Ruan-Tian invariants [RT]). In [T3]-[T5] Taubes proves, on a symplectic 4-manifold with $b^{+}>1$, the equivalence between Seiberg-Witten invariants and Gromov-Taubes invariants. And in the case when $b^{+}=1$, the equivalence holds for all the classes with pairing on all the embedded symplectic -1 spheres bigger than -2 .

Taubes conjectured the equivalence should still hold for the remaining classes in the case of $b^{+}=1$. In this regard, McDuff suggested a modification of the Gromov-Taubes invariants ([M]). Adopting McDuff's modification, we [LL2] are able to give an affirmative answer to Taubes's conjecture for the remaining classes using the blow up formulas for Seiberg-Witten invariants and Gromov-Taubes invariants. 
For symplectic manifolds, the equivalence between the differentiable invariants SW and the symplectic invariants GT has led to many striking consequences to symplectic topology. To broaden the scope of the application of the Seiberg-Witten theory, it is desirable to construct some kind of secondary Seiberg-Witten invariants. It is natural to consider the parametrized Seiberg-Witten theory. This means, instead of a single four manifold, we consider a fibre bundle with a smooth four-manifold as the fibre. Upon fixing a smooth family of fibrewise $\operatorname{Spin}^{c}$ structures and a family of real self-dual two forms, there arises a family of Seiberg-Witten equations, and the space of fibrewise monoples gives rise to various kind of invariants, the family Seiberg-Witten invariants.

This idea of the parametrized Seiberg-Witten theory was suggested by Donaldson in [D2] (see also the recent papers $[\mathrm{K}]$ and [Rub]). In this paper, we will develop the Seiberg-Witten theory in the family setting in some generality. Though we restrict ourselves to the case that the base is a closed oriented manifold, many of the results immediately apply to the case where the base is an oriented manifold with boundary.

A new feature of the family Seiberg-Witten theory is that the chamber structure plays a more prominent role. For the ordinary Seiberg-Witten invariants, only if $b^{+}=1$ they depend on chambers. For the family SeibergWitten invariants, they depend on chambers as long as $b^{+}-1$ is less than or equal to the dimension of the base, and the chamber structure is much more complicated. We will introduce a finite dimensional bundle, the period bundle, and make use of this bundle to classify the set of chambers. In general the chamber structure is very complicated. However, when the dimension of the base is exactly equal to $b^{+}-1$, the chamber structure is simple, it is either $\mathbf{Z}_{2}$ if $b^{+}=1$ or $\mathbf{Z}$ if $b^{+}>1$. We call this case the critical case.

As a pleasant by product of the study of the chamber structure, we can extend the scope of certain homomorphism $Q$ from the homology of the space of cohomologous symplectic forms introduced by Kronheimer [K]. This simple extension is interesting, for example, on $S^{2} \times S^{2}$, it is nontrivial while Kronheimer's original $Q$ is not even defined.

In the cirtical case we extend our techniques in [LL1] to prove a wall crossing formula. The wall crossing formula is universal in the sense that it only depends on the four-manifold, not on the topology of the fibre bundle.

But there are many interesting families with high dimensional bases which fall into the non-critical case $b^{+}-1<\operatorname{dim} B$. We will study the Fulton-MacPherson family, which are built up from the Fulton-MacPherson spaces and derive the corresponding wall crossing formulas. These family are ultimately related to the counting of nodal pseudo-holomorphic curves. In 
fact after certain transformation, the wall crossing formulas are manifestly tied to the enumeration problem in algebraic geometry. This also strongly supports (as a nontrivial example) the general conjecture between the family Seiberg-Witten theory and the family Gromov theory. In fact, Ai-Ko Liu [Liu] proves a blow up formula for parametrized Seiberg-Witten invariants, and when applied to the Fulton-MacPherson familes of Kahler surfaces, it is closely related to the wall crossing formula and has beautiful applications to counting curves in Kahler surfaces.

A symplectic family is a fiber bundle with symplectic four-manifolds as fibers and a smooth family of symplectic forms over the fibers. This is an extremely interesting class of families. Nice examples of such families are hyperkahler families, or more general winding families (defined in section 3), of the K3 surface and the four-torus (the bases are $S^{2}$ ). A first observation is that there is a canonical chamber associated to any symplectic family, the symplectic chamber. The family Seiberg-Witten invariants in the symplectic chamber will be called the family Taubes-Seiberg-Witten invariants. Though the Seiberg-Witten invariants for the K3 surface and the four torus are trivial, the invariants of the winding family are very rich. In fact we realized that the counting of curves on a projective K3 surface is closely related to the invariants (Seiberg-Witten and Gromov) for the hyperkahler family, and Yau and Zaslow's beautiful conjectual generating function of curves on projective K3 [YZ] was one of the motivations to develop the parametrized SeibergWitten theory. Recently, Yau and Zaslow's formula is indeed confirmed via calculation of the Gromov invariants of hyperkahler family of K3 surfaces by Bryan and Leung [BL1].

Because of Taubes's results [T1]-[T5], one naturally expects that the family Seiberg-Witten theory is particularly interesting for symplectic families and the nonvanishing of the family Taubes-Seiberg-Witten invariants will imply the existence of fibrewise pseudo-holomorphic curves. This is indeed the case. However, to set up the family Gromov-Taubes invariants counting fiberwise pseudo-holomorphic curves and push Taubes's equivalence between SW and GT to the family setting, we only have limited success. For a restricted class of families, including the winding families of the four torus and primary Kodaira surfaces, we can give a good definition and prove TSW=GT. These results will appear in [LL3] and be applied to study symplectic manifolds with torsion symplectic canonical classess.

The orgnization of the paper is as follows. In $\S 2$, we introduce the family Seiberg-Witten invariants. In $\S 3$, we introduce the period bundle and study the chamber structure. In $\S 4$, we prove the wall crossing formula in the critical case and study some interesting examples. We also derive the wall 
crossing formula for the Fulton-MacPherson family.

The authors wish to thank S.T. Yau who suggested us to study parametrized Seiberg-Witten theory soon after the birth of the SeibergWitten theory and has given us many valuable ideas and shown great interest. The authors wish to thank C. Taubes and G. Tian for many suggestions and sharing their ideas. The authors wish to thank E. Brown, R. Fintushel, G. Moore, R. Lee, G. Zuckerman for their interest in this work. Both authors are partially supported by NSF and wish to thank the support and hospitality of IAS. Finally the author wish to thank the referee and A. Greenspoon for careful readings and excellent suggestions which greatly improve the presentation of the paper.

\section{Family Seiberg-Witten invariants.}

In this section, we set up the family Seiberg-Witten theory. In the first two subsections, the family Seiberg-Witten equations and the family SeibergWitten invariants are introduced. The last subsection is devoted to the symplectic families.

\subsection{Family Seiberg-Witten equations.}

Let $M$ be an oriented closed 4-manifold. Let $b_{i}$ denote the $i$-th Betti number of $M$ and let $b^{+}$denote the dimension of a maximal subspace, $H^{+}(M ; \mathbf{R}) \subset$ $H^{2}(M ; \mathbf{R})$ where the cup product form is positive.

Let $B$ be an oriented closed manifold and $\mathcal{X}$ be the total space of a fibre bundle with fibre $M$ and base $B$.

Denote the tangent bundle along the fibre by $T(\mathcal{X} / B)$ and the bundles of $i$-forms along the fibre by $\Lambda^{i}$. Choose a metric $G$ on $T(\mathcal{X} / B)$, then it defines a principle $S O(4)$ bundle of frames, $\mathcal{F} r \rightarrow \mathcal{X}$ and two associated bundles, $\Lambda^{+}$of self dual 2-forms along the fibre and $\Lambda^{-}$of anti self dual 2 -forms along the fibre.

For any $b \in B$, denote by $\mathcal{X}_{b}$ the fibre at $b$. It will be understood that for any object defined on the total space $\mathcal{X}$, the object with subscript $b$ will denote the restriction to the fibre $\mathcal{X}_{b}$.

A $\operatorname{Spin}^{c}$ structure $\mathcal{L}$ on $\mathcal{X} / B$ is an equivalence class of lifts of $\mathcal{F} r$ to a principle $\operatorname{Spin}^{c}(4)$ bundle $\mathcal{F}$. Recall the group $\operatorname{Spin}^{c}(4)$ is the group $(S U(2) \times S U(2) \times U(1)) /\{ \pm 1\}$ and the group $S O(4)$ is also the same as the group $(S U(2) \times S U(2)) /\{ \pm 1\}$, and the homomorphism $\operatorname{Spin}^{c}(4) \longrightarrow S O(4)$ is simply the map forgetting the factor $U(1)$. 
Associated with $\mathcal{F}$, there are two $U(2)$ bundle of spinors $\mathcal{S}^{+}$and $\mathcal{S}^{-}$ coming from the two natural homomorphisms from $\operatorname{Spin}^{c}(4)$ to $U(2)=$ $(S U(2) \times U(1)) /\{ \pm 1\} . \mathcal{S}^{+}$is distinguished by the identification of its projective bundle with the unit two sphere bundle of $\Lambda^{+}$. The natural homomorphism $T^{*}(\mathcal{X} / B) \otimes \mathcal{S}^{+} \longrightarrow \mathcal{S}^{-}$defines the Clifford multiplication. And the adjoint of Clifford multiplication endmorphism defines a canonical homomorphism

$$
\tau: \text { End }\left(\mathcal{S}^{+}\right) \longrightarrow \Lambda^{+} \otimes \mathbf{C} .
$$

Let $L=\operatorname{det}\left(\mathcal{S}^{+}\right) . \quad L$ is viewed as a family of $U(1)$ bundles over $B$. Given a smooth family of $U(1)$ connections $A=\left\{A_{b}\right\}$ on $L$, combined with the family of Levi-Civita connections on $\mathcal{F} r$, it defines a smooth family of covariant differentiation on $\mathcal{S}^{+}$still denoted by $A=\left\{A_{b}\right\}$. Let $\psi$ be a section of $\mathcal{S}^{+}$, then it is naturally viewed as a smooth family of sections of $\mathcal{S}_{b}^{+}$. Denote $\mathcal{C}_{b}$ the space of pairs $\left(A_{b}, \psi_{b}\right)$ where $A_{b}$ is a $U(1)$-connection on $L_{b}$ and $\psi_{b}$ is a section of $\mathcal{S}_{b}^{+} . \mathcal{C}_{b}$ is an affine Frechet manifold modelled on $i \Lambda_{b}^{1} \times C^{\infty}\left(\mathcal{S}_{b}^{+}\right)\left(\Lambda_{b}^{1}\right.$ is the space of smooth 1-forms on $\left.\mathcal{X}_{b}\right)$. Then the configuration space $\mathcal{C}$ is the space of pairs $(A, \psi)$. It is just the space of sections of the infinite dimensional affine bundle over $B$ with fibre $\mathcal{C}_{b}$. Call an element $(A, \psi) \in \mathcal{C}$ irreducible if $\psi_{b}$ is not identically zero for any $b$. Denote $\mathcal{C}^{*}$ the irreducible part of $\mathcal{C}$.

The unperturbed Seiberg-Witten equations on $\mathcal{X} / B$ are equations for $(A, \psi) \in \mathcal{C}:$

$$
\begin{aligned}
D_{A_{b}} \psi_{b} & =0 \\
P_{b}^{+} F_{A_{b}} & =\frac{1}{4} \tau\left(\psi_{b} \otimes \psi_{b}^{*}\right) .
\end{aligned}
$$

In the first equation, $D_{A_{b}}: \Gamma\left(\mathcal{S}_{b}^{+}\right) \longrightarrow \Gamma\left(\mathcal{S}_{b}^{-}\right)$is the Dirac operator, a first order differential operator defined using the Clifford multiplication and the covariant differentiation $A_{b}$ on $\mathcal{S}_{b}^{+}$. In the second equation, $P_{b}^{+}: \Lambda_{b}^{2} \longrightarrow \Lambda_{b}^{+}$ is the orthogonal projection and $F_{A_{b}}$ is the curvature two form of $A_{b}$.

Let $\mu \in \Lambda^{+}$, then $\mu_{b}$ defines a real valued self dual two form on $\mathcal{X}_{b}$. It is quite useful to consider perturbations which are of the form

$$
\begin{aligned}
D_{A_{b}} \psi_{b} & =0 \\
P_{b}^{+} F_{A_{b}} & =\frac{1}{4} \tau\left(\psi_{b} \otimes \psi_{b}^{*}\right)+i \mu_{b} .
\end{aligned}
$$

The parameter space for the family Seiberg-Witten equations is thus the following subspace of the product $\mathcal{I} \times i \Lambda^{2}$ :

$$
\Gamma=\left\{(G, \mu) \mid \text { for each } b, \mu_{b} \text { is self-dual with respect to } G_{b}\right\}
$$


where $\mathcal{I}$ is the space of metrics on $T(\mathcal{X} / B) . \mathcal{I}$ is an infinite dimensional fibre bundle over $B$ whose fibre at $b$ is the space of Riemannian metrics on $\mathcal{X}_{b}$ (a Frechet manifold).

$\Gamma$ has an alternative description which is often more convenient. Denote by $\tilde{\mathcal{P}}$ the infinite dimensional bundle over $B$ whose fibre over $b$ is the space of pairs $\left(G_{b}, \mu_{b}\right)$ where $G_{b}$ is a metric on $\mathcal{X}_{b}$ and $\mu_{b}$ is a real valued two form and self-dual with respect to $G_{b}$. Then $\Gamma$ is just the space of sections of $\tilde{\mathcal{P}}$. To study $\Gamma$, we introduce $\overline{\mathcal{P}}$, a finite dimensional bundle over $B$ whose fibre over $b$ is the product of the real Grassmannian $\operatorname{Gr}\left(H_{b}, b^{+}\right)$and the vector space $H_{b}$, where $H_{b}$ is $H^{2}\left(\mathcal{X}_{b} ; \mathbf{R}\right)$. There is a natural map $r_{\mathcal{L}}$ from $\tilde{\mathcal{P}}$ to $\overline{\mathcal{P}}$ sending each pair $\left(G_{b}, \mu_{b}\right)$ to the pair $\left(\left[\mathcal{H}^{+}\right], \mathcal{H}\left(2 \pi c_{1}(L)-\mu_{b}\right)\right)$ where $\left[\mathcal{H}^{+}\right]$is the point in the real Grassmannian given by the $b^{+}$dimensional subspace of self-dual harmonic two forms and $\mathcal{H}\left(2 \pi c_{1}(L)-\mu_{b}\right)$ is the harmonic projection of $2 \pi c_{1}(L)-\mu_{b}$, both with respect to the metric $G_{b}$. The next section will be devoted to the homotopy classes of the sections of the period bundle $\mathcal{P}$.

Given any pair $(G, \mu) \in \underset{\tilde{M}}{ }$, denote the space of solutions to $(2.2)$ by $\tilde{\mathcal{M}}$ and its irreducible part by $\tilde{\mathcal{M}}^{*}$.

The gauge group is replaced by the bundle of groups $\mathcal{G}$ over $B$, whose fiber over $b$ is the group $\mathcal{G}_{b}=C^{\infty}\left(\mathcal{X}_{b}, U(1)\right)$. $\mathcal{G}_{b}$ acts smoothly on $\mathcal{C}_{b}$ by sending a map $g_{b}$ and a pair $\left(A_{b}, \psi_{b}\right)$ to $\left(A_{b}+2 g_{b} d g_{b}^{-1}, g_{b} \psi_{b}\right)$ and the action is free on $\mathcal{C}_{b}^{*}$. This action naturally extends to a smooth map from $\mathcal{G} \times{ }_{B} \mathcal{C}$ to $\mathcal{C}$ which defines a $\mathcal{G}$ action on $\mathcal{C}$ (we abuse language here and elsewhere in this paper, though $\mathcal{G}$ is not really a group, we will say that it acts on $\mathcal{C})$. And the space of the orbits of this action, denoted by $\mathcal{C} / \mathcal{G}$, is given the quotient topology. Just as in the case of the ordinary Seiberg-Witten equations, $\mathcal{G}$ preserves $\tilde{\mathcal{M}}$ and $\tilde{\mathcal{M}}^{*}$, and the quotients are called $\mathcal{M}$ and $\mathcal{M}^{*}$ respectively with the subspace topology inherited from $\mathcal{C} / \mathcal{G}$.

For any $(G, \mu) \in \Gamma, \mathcal{M}$ is always compact. Since $B$ is compact, the Weizenbock formula still gives an uniform bound of the $C^{0}$ norm of $\psi_{b}$ of any solution $\left(A_{b}, \psi_{b}\right)$ to $(2.2)$. From it, we can derive the uniform bound of the $C^{\infty}$ norm of solutions to (2.2) up to gauge, and the compactness follows.

Let $\left(A_{b}, \psi_{b}\right)$ be a solution to $(2.2)$. If we linearize the $\mathrm{SW}$ equations at $\left(A_{b}, \psi_{b}\right)$ and take into account the gauge group action, we get a linear Fredholm operator,

$$
\mathcal{D}: T_{b} B \oplus i \Lambda_{b}^{1} \oplus \Gamma\left(\mathcal{S}_{b}^{+}\right) \longrightarrow i \Lambda_{b}^{+} \oplus \Gamma\left(\mathcal{S}_{b}^{-}\right) \oplus i \Lambda_{b}^{0}
$$

$\mathcal{D}$ is Fredholm of index

$$
d=d(\mathcal{L})=\operatorname{dim} B+\frac{1}{4}\left[c_{1}(L)^{2}-(2 e(M)+3 \sigma(M))\right]
$$


where $e(M)$ is the Euler characteristic of $M$ and $\sigma(M)$ is the signature of $M$.

By the Smale-Sard theorem, for generic $(G, \mu) \in \Gamma$, here generic means a Baire set, $\mathcal{D}$ is surjective at all the irreducible solutions, so $\mathcal{M}^{*}$ is a smooth manifold of dimension $d$ as in (2.3).

Given a $\operatorname{Spin}^{c}$ structure $\mathcal{L}$, we define a subset $\mathcal{W} A(\mathcal{L})$ in $\Gamma$ which we will call the " $\mathcal{L}$-wall" in $\Gamma$. A pair $(G, \mu)$ is in $\mathcal{W} A(\mathcal{L})$ if for some $b, 2 \pi c_{1}\left(L_{b}\right)-\mu_{b}$ is anti self dual with respect to $G_{b}$. For any pair $(G, \mu) \in \Gamma-\mathcal{W} A(\mathcal{L}), \tilde{\mathcal{M}}$ contains no reducible solutions, so $\tilde{\mathcal{M}}=\overline{\mathcal{M}^{*}}$.

From the second description of $\Gamma$, it is not hard to see that $\mathcal{W} A(\mathcal{L})$ is a codimension $\max \left(b^{+}-\operatorname{dim} B, 0\right)$ submanifold in $\Gamma$. In particular, if $b^{+}-1 \geq \operatorname{dim} B$, for a generic pair $(G, \mu)$ (here generic means an open set off a submanifold of positive codimension), there are no reducible solutions. Even when $b^{+} \leq \operatorname{dim} B$, as long as $b^{+} \neq 0$, it is not hard to see any pair $(G, \mu)$ with sufficient large $\mu$ does not lie in $\mathcal{W} A$, thus $\Gamma-\mathcal{W} A(\mathcal{L})$ still contains a nonempty open set. Call each connected component of $\Gamma-\mathcal{W} A(\mathcal{L})$ a $\mathcal{L}$-chamber.

A choice of an orientation of the real line $\operatorname{det}^{+}=\operatorname{det}\left(H^{0}(M ; \mathbf{R}) \otimes\right.$ $H^{1}(M ; \mathbf{R}) \otimes H^{+}(M ; \mathbf{R}) \otimes H^{\operatorname{dim} B}(B ; \mathbf{R})$ serves to orient $\mathcal{M}$.

Hence there are always pairs $(G, \mu) \in \Gamma$ such that $\mathcal{M}=\mathcal{M}^{*}$ and $\mathcal{M}^{*}$ is a closed manifold of dimension $d$ as in (2.3) in the space $\mathcal{C}^{*} / \mathcal{G}$, and after fixing an orientation of the determinant line $\operatorname{det}^{+}$, it represents a homology class $\left[\mathcal{M}^{*}\right]$ in $H_{d}\left(\mathcal{C}^{*} / \mathcal{G} ; \mathbf{Z}\right)$. If $b^{+}-1 \geq \operatorname{dim} B$, such pairs $(G, \mu) \in \Gamma$ are generic.

\subsection{Family Seiberg-Witten invariants.}

Denote the set of $\operatorname{Spin}^{c}$ structures on $\mathcal{X} / B$ by $\mathcal{S} P$. Although the definition of $\mathcal{S} P$ requires a choice of metric on $T(\mathcal{X} / B)$, there is a natural identification between such sets defined by any two metrics. Thus, the set $\mathcal{S} P$ only depends on the fibre bundle $\mathcal{X} / B$ and is an affine space modelled on $H^{2}(\mathcal{X} ; \mathbf{Z})$.

Given an element $\mathcal{L} \in \mathcal{S} P$, the definition of the family SW invariants of $\mathcal{L}$ depends on a cohomology class $\Theta$ on the space $\mathcal{C}^{*} / \mathcal{G}$ and a choice of parameter $(G, \mu) \in \Gamma$.

Fix a pair $(G, \mu)$ such that $\mathcal{M}$ is a closed manifold of dimension $d=$ $d(\mathcal{L})$ as in (2.3). Fix an orientation of the determinant line. Choose $\Theta \in$ $H^{l}\left(\mathcal{C}^{*} / \mathcal{G} ; \mathbf{Z}\right)$, the Seiberg-Witten invariant associated to $\Theta$ is defined to be

$$
S W(\mathcal{X} / B, \mathcal{L}, \Theta)=\langle\Theta,[\mathcal{M}]\rangle
$$

if $l=d$, and zero otherwise, where $\langle-,-\rangle$ is the pairing between cohomology 
and homology on the space $\mathcal{C}^{*} / \mathcal{G}$. Sometimes we will drop $\mathcal{X} / B$ or $\mathcal{L}$ in the notation when there is no confusion.

If $b^{+}-1>\operatorname{dim} B$, given a $\operatorname{Spin}^{c}$ structure $\mathcal{L}$ on $\mathcal{X} / B$ then the SW invariants of $\mathcal{L}$ is defined for generic pair $(G, \mu) \in \Gamma-\mathcal{W} A(\mathcal{L})$. And since $\Gamma-\mathcal{W} A(\mathcal{L})$ is path connected, from the standard cobordism arguments, the value of the Seiberg-Witten invariant is independent of the choice of the parameter. This is analogous to the invariance of the ordinary SeibergWitten invariant in the case $b^{+}$is greater than one. Hence we have the following theorem:

Theorem 2.1. Let $M$ be a closed oriented 4-manifold and $B$ be a closed oriented manifold. Let $\mathcal{X}$ be a fibre bundle with fibre $M$ and base $B, \mathcal{L}$ be a Spin $^{c}$ structure on $\mathcal{X} / B$ and $\Theta$ be a cohomology class in $H^{*}\left(\mathcal{C}^{*} / \mathcal{G} ; \mathbf{Z}\right)$. Fix an orientation of $\operatorname{det}^{+}$. If $b^{+}-1>\operatorname{dim} B$, then $S W(\mathcal{X} / B, \mathcal{L}, \Theta)$ is independent of the choice of generic $(G, \mu)$, and hence is a differentiable invariant of the fiber bundle. Furthermore, if $f$ is a self diffeomorphim of $\mathcal{X}$ preserving the fibers, then

$$
S W(\mathcal{X} / B, \mathcal{L}, \Theta)= \pm S W\left(\mathcal{X} / B, f^{*} \mathcal{L}, f^{*} \Theta\right) .
$$

In the case $b^{+}-1 \leq \operatorname{dim} B$, the preceding theorem does not hold. However, by the standard arguments, the following conclusion still holds:

Theorem 2.2. Let $M$ be a closed oriented 4-manifold and $B$ a closed oriented manifold. Let $\mathcal{X}$ be a fibre bundle with fibre $M$ and base $B, \mathcal{L}$ be a Spin ${ }^{c}$ structure on $\mathcal{X} / B$ and $\Theta$ be a cohomology class in $H^{*}(\mathcal{C} / \mathcal{G} ; \mathbf{Z})$. Fix an orientation of $\operatorname{det}^{+}$. Suppose $b^{+}-1 \leq \operatorname{dim} B$ and let $\Gamma_{c}$ be a $\mathcal{L}$-chamber. Then

1. if $b^{+}=\operatorname{dim} B+1, S W(\mathcal{X} / B, \mathcal{L}, \Theta, \mathcal{C})$ is defined for generic pairs $(G, \mu) \in \Gamma_{c}$ and is independent of the choice of $(G, \mu)$.

2. if $0<b^{+} \leq \operatorname{dim} B, S W(\mathcal{X} / B, \mathcal{L}, \Theta, c)$ is defined for some pairs $(G, \mu) \in \Gamma_{c}$ and is again independent of the choice of $(G, \mu)$.

With Theorem 2.2 understood, it is important to pin down the dependence on chambers. This will be the content of the next two sections.

In the remaining part of this subsection, we will describe how to construct some interesting cohomology classes on the infinite dimensional object $\mathcal{C}^{*} / \mathcal{G}$.

The first observation is that it is homotopic to a $C P^{\infty} \times T^{b_{1}}$ bundle over $B$. Any cohomology class of $B$ pulls back to a cohomology class of $\mathcal{C}^{*} / \mathcal{G}$. If $\Theta$ can be factored by such a class with degree $l$, then we call $\Theta$ has weight $l$. There is a distinguished class $[B]^{\wedge}$ in $H^{\operatorname{dim} B}\left(\mathcal{C}^{*} / \mathcal{G} ; \mathbf{Z}\right)$ which is 
the pull back of the fundamental cohomology class of $B$. If $\Theta$ is factored by $[B]^{\wedge}$, then the family Seiberg-Witten invariant becomes an ordinary SeibergWitten invariant. Similarly, if $\Theta$ has weight $l$, then the invariant reduce to an invariant on a codimension $l$ family.

Another observation is that $\mathcal{G}$ naturally acts on the the complex line bundle $L$ over $\mathcal{X}$. The diagonal action on the product $L \times \mathcal{C}^{*}$ defines a complex line bundle $L \times \mathcal{G} \mathcal{C}^{*}$ over $\mathcal{X} \times\left(\mathcal{C}^{*} / \mathcal{G}\right)$. Denote the first Chern class of this line bundle by $u$ and the restriction of $u$ to $\mathcal{C}^{*} / \mathcal{G}$ by $H . H$ will be called the hyperplane class.

Via slant product with $u$, we get a map

$$
\mu: H_{i}(\mathcal{X} ; \mathbf{Z}) \longrightarrow H^{2-i}\left(\mathcal{C}^{*} / \mathcal{G} ; \mathbf{Z}\right) .
$$

Pick homology classes $\zeta_{1}, \ldots, \zeta_{l}$ in $H_{*}(\mathcal{X} ; \mathbf{Z})$, we can define

$$
S W\left(\mathcal{X} / B, \mathcal{L}, \mu\left(\zeta_{1}\right) \cdots \mu\left(\zeta_{l}\right)\right)=S W\left(\mathcal{X} / B, \mathcal{L}, \mu\left(\zeta_{1}\right) \wedge \cdots \wedge \mu\left(\zeta_{l}\right)\right) .
$$

A particular important invariant, the pure invariant, is given in the following definition.

Definition 2.3 (pure invariant). When the dimension of $\mathcal{M}$ is even, we define the pure invariant to be

$$
S W(H)=\int_{\mathcal{M}} H^{\operatorname{dim} \mathcal{M} / 2} .
$$

Set $U=H \cup[B]^{\wedge}$. Via slant product with $U$, we get a map

$$
\bar{\mu}: H_{i}(\mathcal{X} ; \mathbf{Z}) \longrightarrow H^{\operatorname{dim} B+2-i}\left(\mathcal{C}^{*} / \mathcal{G} ; \mathbf{Z}\right) .
$$

Pick homology classes $\xi_{1}, \ldots, \xi_{l}$ in $H_{*}(\mathcal{X} ; \mathbf{Z})$, we can define

$$
S W\left(\mathcal{X} / B, \mathcal{L}, \bar{\mu}\left(\xi_{1}\right), \ldots, \bar{\mu}\left(\xi_{l}\right)\right) .
$$

It counts the number of fiberwise monoples whose zero set intersect each of the submanifolds $V_{i}$, where $V_{i}$ represents $\xi_{i}$.

When $\mathcal{X}$ is a product bundle, $\mu$ and $\bar{\mu}$ are simply related by

$$
\mu(\zeta)=\bar{\mu}(\zeta \times[B])
$$

Example 2.4 (section invariants and circle section invariants). Suppose $\mathcal{X}$ has sections. Given any section $s: B \longrightarrow \mathcal{X}$, we can define a 
based gauge group $\mathcal{G}(s) \subset \mathcal{G}$ consisting of maps sending $s(B)$ to the identity. Define

$$
\mathcal{C}(s)=\mathcal{C} / \mathcal{G}(s) \text { and } \quad \mathcal{C}^{*}(s)=\mathcal{C}^{*} / \mathcal{G}(s) .
$$

Then $\mathcal{C}^{*}(s)$ is a principle $S^{1}$ bundle over $\mathcal{C}^{*} / \mathcal{G}$ and its euler class $e(s)$ is in $H^{2}\left(\mathcal{C}^{*} / \mathcal{G} ; \mathbf{Z}\right)$. Notice a section $s$ determines a homology class in $H_{*}(\mathcal{X} ; \mathbf{Z})$, and $e(s)$ is simply the slant product of this homology class with $U$. If $d$ is an even integer, then after picking $d / 2$ number of sections, $s_{1}, \ldots, s_{d / 2}$, the cohomology class $\prod_{i=1}^{d / 2} e\left(s_{i}\right)$ is of degree $d$. We can define the corresponding Seiberg-Witten invariant $S W\left(\mathcal{X} / B, \mathcal{L}, s_{1}, s_{2}, \ldots, s_{d / 2}\right)$. This invariant only depends on $\left[s_{i}\right], i=1, \ldots, d / 2$, the homotopy classes of $s_{i}$, as the corresponding cohomology classes depend on the homotopy classes only. A geometric interpretation of this invariant is that it counts the number of fiberwise monoples vanishing along these sections.

Similarly, if $\mathcal{X}$ allows sections of circles, each circle section defines a homology class of $\mathcal{X}$, and induces a cohomology class of $\mathcal{C}^{*} / \mathcal{G}$ via the slant product. Thus, given $d$ circle sections $\gamma_{1}, \ldots, \gamma_{d}$, we can define the SW invariant $S W\left(\mathcal{X} / B, \mathcal{L}, \gamma_{1}, \ldots, \gamma_{d}\right)$ which only depend on the isotopy classes of the circle sections. Geometrically, this invariant counts the number of fiberwise monoples which are not nowhere vanishing along each of the circle sections. More generally, given an integer $p$ such that $d-p$ is a nonnegative even integer, and $p$ circle sections $\gamma_{1}, \ldots, \gamma_{p}$ and $(d-p) / 2$ sections $s_{1}, \ldots, s_{(d-p) / 2}$, we can define the Seiberg-Witten invariant $S W\left(\mathcal{X} / B, \mathcal{L}, \gamma_{1}, \ldots, \gamma_{p}, s_{1}, \ldots, s_{(d-p) / 2}\right)$.

Remark 2.5. Donaldson, in his beautiful survey article [D2], suggested the possibility to construct the family Seiberg-Witten invariants. He further suggested that these invariants, should be viewed as cohomology classes of $\operatorname{Bdiff}(M)$, the classifying space of the group of diffeomorphisms of $M$.

\subsection{Seiberg-Witten invariants on symplectic families.}

In this subsection, we describe the special features of the family SeibergWitten invariants on sympletic families. The readers should consult [T1].

Let $\mathcal{X}$ be a fibre bundle of a four manifold over $B$ and $\omega$ be a two form on $\mathcal{X}$ whose restriction to each fibre is a symplectic form. Each fiber $\mathcal{X}_{b}$ is oriented by $\omega_{b} \wedge \omega_{b}$.

Fix a metric $G$ such that $\omega_{b}$ is self-dual with respect to $G_{b}$. $G$ can be further normalized such that $\omega_{b}$ has length $\sqrt{2}$. Such a family metric is called a $\omega$ compatible metric. $\omega$ and a $\omega$ compatible metric determine a smooth 
family of compatible almost complex structures $J$ on the fibers, hence a complex structure (still denoted by $J$ ) of the bundle $T(\mathcal{X} / B)$. Taking the complex determinant, one obtains a complex line bundles $K$ over $\mathcal{X}$, whose restriction to each fibre $\mathcal{X}_{b}$ is given by $\operatorname{det}\left(T^{0,1} \mathcal{X}_{b}\right)$.

There is a canonical $\operatorname{Spin}^{c}$ structure with the associated bundles $\mathcal{S}^{+}$and $\mathcal{S}^{-}$naturally isomorphic to $\mid \oplus K^{-1}$ and $\Lambda^{0,1}=\Lambda^{0,1}(T(\mathcal{X} / B))$, where $\mid$ is the trivial complex bundle over $\mathcal{X}$. The splitting of $\mathcal{S}^{+}$is induced by the Clifford action of $\omega$, which has eigenvalue $-2 i$ on the trivial summand $\mid$ and eigenvalue $2 i$ on the $K^{-1}$ summand.

This canonical $\mathrm{Spin}^{c}$ structure induces a natural identification between the set $\mathcal{S} P$ and $H^{2}(\mathcal{X} ; \mathbf{Z})$. Under this identification, a class $e \in H^{2}(\mathcal{X} ; \mathbf{Z})$ is sent to the $\operatorname{Spin}^{c}$ structure whose $\mathcal{S}^{ \pm}$bundles are given by

$$
\mathcal{S}^{+}=E \oplus\left(K^{-1} \otimes E\right) \quad \text { and } \quad \mathcal{S}^{-}=\Lambda^{0,1} \otimes E .
$$

where $E$ is a complex line bundle over $\mathcal{X}$ whose first Chern class is isomorphic to $e$.

There is a natural orientation for the line $\operatorname{det}^{+}$provided an orientation of $B$ is fixed. This is because the half-selfdual complex on $M$ is within a relative compact perturbation of a complex linear complex arising of a compatible almost complex structure.

To introduce the family version of Taubes's perturbation of the SW equations, it requires the introduction of a smooth family of canonical connections $A^{0}$ (up to the gauge action) on $K_{b}^{-1}$. A family of connections on $K^{-1}$, coupled with the family of Levi-Civita connections gives rise to a family of covariant derivatives, $\nabla_{A}$, on $\mathcal{S}^{+}$. Through restriciton and projection, $\nabla_{A}$ defines a family of covariant derivatives, $\nabla_{A}$, on the trivial summand $\mid$. The family $A^{0}$ is characterized by the property that the corresponding family of covariant derivatives, $\nabla_{A^{0}}$, admits a family of non-trivial covariantly constant sections, $u^{0}$. In the subsequent discussions, $u^{0}$ will be normalized such that $u_{b}^{0}$ has norm one.

Let $\mathcal{L}$ be a $\operatorname{Spin}^{c}$ structure on $\mathcal{X} / B$ specified by a complex line bundle $E$ over $\mathcal{X}$ under the aforementioned identification. Recall, in the family setting, the SW equations are equtions for pairs $\left(A_{b}, \psi_{b}\right)$, where $A_{b}$ is a connection on the complex line bundle $L_{b}=\operatorname{det} \mathcal{S}_{b}^{+}$and $\psi_{b}$ is a section of $\mathcal{S}_{b}^{+}$. Remark that each line bundle $L_{b}$ is naturally the restriction of the complex line bundle $L=\operatorname{det} \mathcal{S}^{+}$to the fiber $\mathcal{X}_{b}$. From (2.5), $L_{b}$ is $K_{b}^{-1} \otimes E_{b}$. Thus, with $A^{0}$ fixed, a connection $A_{b}$ on $L_{b}$ is written as $A_{b}=A_{b}^{0}+2 a_{b}$, where $a_{b}$ is a connection on the complex line bundle $E_{b}$.

Following Taubes, we choose the one parameter family (parametrized by 
$r$ ) of the perturbation two forms $\mu$ by

$$
\mu_{b}=\frac{-r}{4} \omega-i P_{+} F_{A_{b}^{0}} .
$$

It is useful to rewrite the spinor corresponding to the splitting and the parameter $r$ as

$$
\psi_{b}=r^{1 / 2} \cdot\left(\alpha_{b} u_{b}^{0}+\beta_{b}\right) .
$$

Now the equations read

$$
\begin{gathered}
\sigma\left(u^{0} \otimes \nabla_{a_{b}} \alpha_{b}\right)+D_{A_{b}} \beta_{b}=0 \\
P_{+} F_{a}=-\frac{i}{r} \cdot\left(1-\left|\alpha_{b}\right|^{2}+\left|\beta_{b}\right|^{2}\right) \cdot \omega_{b}+\frac{i r}{4}\left(\alpha_{b} \beta_{b}^{*}+\alpha_{b}^{*} \beta_{b}\right) .
\end{gathered}
$$

Here, $\alpha_{b} \beta_{b}^{*}$ and $\alpha_{b}^{*} \beta_{b}$, being respective sections of $K_{b}$ and $K_{b}^{-1}$, are identified as sections of $\Lambda_{b}^{+} \otimes \mathbf{C}$.

Notice that there is a canonical chamber, called the Taubes chamber, for any $\operatorname{Spin}^{c}$ structure $\mathcal{L}$ on a symplectic family. On each fiber $\mathcal{X}_{b}$, when $r$ is sufficiently large, $2 \pi c_{1}(L)-\mu_{b}$ has positive square due to the term $r^{2} \omega \wedge \omega / 16$. So no reducible solutions can possibly occur for all large $r$. Since $B$ is compact, there is a uniform constant such that $2 \pi c_{1}(L)-\mu_{b}$ has positive square for all $b$ once $r$ is greater than that constant. This means that for all large $r$, the pair $(G, \mu)$ with $\mu$ given by (2.6) lie in the same $\mathcal{L}$ chamber, and this chamber will be called the Taubes chamber. Notice this chamber only depends on the family of symplectic forms through its deformation class: If $\omega$ and $\omega^{\prime}$ are two familes of symplectic forms on a fibre bundle, and there is a path of families of symplectic two forms connecting $\omega$ and $\omega^{\prime}$, then the Taubes's chambers for these two families are the same. Finally, let us give a definition.

Definition 2.6. Given a symplectic family, the Seiberg-Witten invariants in the Taubes chamber are called the Taubes-Seiberg-Witten invariants.

\section{The period bundle and the chamber structure.}

When $0 \leq b^{+}-1 \leq \operatorname{dim} B, \Gamma-\mathcal{W} A(\mathcal{L})$ may have more than one component, i.e., the number of $\mathcal{L}$ chambers may be greater than one. When $B$ is a point, we are back to the ordinary Seiberg-Witten theory in the case $b^{+}=1$, and the chamber structure is well understood, there are two $\mathcal{L}$ chambers. For general $B$, the chamber structure is much more complicated. In this section 
we will discuss the chamber structure when $0 \leq b^{+}-1 \leq \operatorname{dim} B$ and give a classification in cohomological terms. We will derive the wall crossing formula in the next section.

In the first subsection, we will reduce this problem to a finite dimensional bundle $\mathcal{P}$, the period bundle, and study the topology of this bundle. In the next subsection, we will give the classification.

\subsection{Reduction to the period bundle $\mathcal{P}$.}

The parameter space $\Gamma$ is the space of sections of the infinite dimensional bundle $\tilde{\mathcal{P}}$. Fix a $\operatorname{Spin}^{c}$ structure $\mathcal{L}$ with the associated complex line bundle $L$, there is a natural map $\operatorname{pr}_{\mathcal{L}}$ from $\tilde{\mathcal{P}}$ to $\overline{\mathcal{P}}$ sending the pair $\left(G_{b}, \mu_{b}\right)$ to the pair $\left(\left[\mathcal{H}_{b}^{+}\right], \mathcal{H}\left(2 \pi c_{1}\left(L_{b}\right)-\mu_{b}\right)\right)$ where $\left[\mathcal{H}_{b}^{+}\right]$is the point in the real Grassmannian given by the $b^{+}$dimensional subspace of self-daul harmonic two forms and $\mathcal{H}_{b}\left(2 \pi c_{1}\left(L_{b}\right)-\mu_{b}\right)$ is the harmonic projection of $2 \pi c_{1}\left(L_{b}\right)-\mu_{b}$, both with respect to the metric $G_{b}$.

The map $p r_{\mathcal{L}}$ is not surjective, it surjects onto an open subbundle. In that regard, we introduce the following definition.

Definition 3.1 (Self Dual Grassmannian). Let $V$ be a vector space with a nondegenerate quadratic form defined over $R$ with signature $(p, q)$. All the positive definite $\mathrm{p}$ planes in $V$ form an open subset of the p plane Grassmanian in $V$. It will be called the self-dual Grassmannian, and denoted by $G_{p}^{+}(V)$.

Let $\mathcal{P}^{+}$denote the subbundle of $\overline{\mathcal{P}}$ whose fiber over $b$ is $G_{b^{+}}^{+}\left(H^{2}\left(\mathcal{X}_{b} ; \mathbf{R}\right)\right) \times$ $H^{2}\left(\mathcal{X}_{b} ; \mathbf{R}\right)$. We first give a simple observation.

Lemma 3.2. The map $p r_{\mathcal{L}}$ surjects onto $\mathcal{P}^{+}$and is regular everywhere. Moreover, the preimage of every point is contractible.

These properties are not hard to prove. We just mention two useful facts here. The first fact is that the space of metrics fibers over the space of conformal structures with contractible fibers, the space of positive functions. The second fact is that a conformal structure is specified by a real three dimensional positive subbundles, and relative to a fixed Riemannian metric, the space of conformal structures is identified with bundle maps

$$
\nu: \Lambda^{+} \longrightarrow \Lambda^{-},|\nu(\eta)|<|\eta|
$$


The point is now the study of the parameter space $\Gamma$, which is the space of the sections of $\tilde{\mathcal{P}}$, is reduced to the study of the space of sections of the finite dimensional bundle $\mathcal{P}^{+}$.

Given a $\operatorname{Spin}^{c}$ structure $\mathcal{L}$, what we really want to understand is the space $\Gamma-\mathcal{W} A(\mathcal{L})$. For this purpose, we need to introduce the period bundle, which is a subbundle of $\mathcal{P}^{+}$.

Let us first assume that $B$ is a point. Then, a pair $(G, \mu)$ lies in $\mathcal{W} A(\mathcal{L})$ if $P_{+} F_{A}-i \mu=0$ has a solution. But this is equivalent to the following: inside $C^{\infty}\left(\Lambda^{+}\right)$, the affine subspace $\left\{A \longrightarrow \frac{1}{i} F_{A}-\mu\right\}$ passes through the origin, and thus coincide with the linear subspace $d C^{\infty}\left(\Lambda^{1}\right)$. This condition is also equivalent to $\mathcal{H}\left(2 \pi c_{1}(L)-\mu\right)$ being perpendicular to $\mathcal{H}^{+}$. This simple observation enables us to describe explicitly the image of $\Gamma-\mathcal{W} A(\mathcal{L})$ under the map $p r_{\mathcal{L}}$.

As $B$ is a point, $\tilde{\mathcal{P}}$ is itself the space $\Gamma$ and $\mathcal{P}^{+}$is simply a product $G_{p}^{+}(V) \times V$ where $V=H^{2}(M ; \mathbf{R})$. The question we are interested in is what kind of positive p plane will be perpendicular to a chosen element $l$ in the vector space $V$. In that regard, we introduce a subspace of $\mathcal{P}^{+}, \mathcal{P}$, consisting of the pairs $(W, l)$ in $\mathcal{P}^{+}$such that $l$ is not perpendicular to $W . \mathcal{P}$ is called the period space of $V$. Under the map $\operatorname{pr}_{\mathcal{L}}, \Gamma-\mathcal{W} A(\mathcal{L})$ and $\mathcal{W} A(\mathcal{L})$ are sent onto $\mathcal{P}$ and $\mathcal{P}^{+}-\mathcal{P}$ respectively. As remarked before, the map $\operatorname{pr}_{\mathcal{L}}$ is regular everywhere with contractible preimages, therefore $\Gamma-\mathcal{W} A(\mathcal{L})$ is homotopic to $\mathcal{P}$.

To study the topology of $\mathcal{P}$, it is necessary to understand $G_{p}^{+}(V)$ first. If $p$ is nonzero, $G_{p}^{+}(V)$ is actually contractible, this follows from the the fact that $G_{p}^{+}(V)$ can be identified with $S O(p, q) / S O(p) \times S O(q)$ which is a homogeneous space formed by maximal compact subgroup quotient. Another way to check it is by observing that the projection from one positive $p$ plane to another one induces an isomorphism between these two vector spaces. Fixing a base positive $p$ plane and projecting every other positive $p$ planes to the fixed one induces a smooth function on $G_{p}^{+}(V)$ which has a unique maximum at the base point. This can be done by comparing the volume form (absolute value) of a positive $p$ plane with that of the base point. The upward gradient flow associated to this function produces a homotopy which shrinks $G_{p}^{+}(V)$ to a point.

The period space, viewed as an open submanifold in $G_{p}^{+}(V) \times V$, can be characterised as the complement of the vanishing locus of certain explicit map from $G_{p}^{+}(V) \times V$ to $R^{p}$. The map is defined by first choosing a base point in $G_{p}^{+}(V)$, then projecting the specified $p$ plane to the other positive $p$ plane. As we have explained, the projection map is always nondegenerate, thus a frame in the base $p$ plane will induce a smooth family of frames. Now 
we are ready to define the map from $G_{p}^{+}(V) \times V$ to $\mathbf{R}^{p}$. Picking a point in $G_{p}^{+}(V)$, which is just a positive $p$ plane, and an element in $V$, the pairing between the attached frame in the $p$ plane and the element in $V$ gives us $p$ elements in $\mathbf{R}$, thus it determines a map $c$ from $G_{p}^{+}(V) \times V$ to $\mathbf{R}^{p}$. The pairs $(W, l)$ in the preimage of $0 \in \mathbf{R}^{p}$ are characterized by the property that the $p$ plane $W$ is perpendicular to $l$ in $V$. Deleting these points from $G_{p}^{+}(V) \times V$ results in the period space. This description of the period space readily leads to the following proposition.

Proposition 3.3. Let $V$ be a vector space with a quadratic form of signature $(p, q)$ defined over $\mathbf{R}$. The period space associated to the vector space $V$ is homotopic equivalent to $S^{p-1}$.

In fact it is easy to show that the map $c$ is regular everywhere. Thus the preimage of every point in $\mathbf{R}^{p}$ is diffeomorphic to each other. Therefore, the period space has a structure of a fiber bundle with $\mathbf{R}^{p}-\{0\}$ as base. To show that the period space is homotopic to $S^{p-1}$, it is sufficient to show that every fiber is contractible. It is an easy exercise that we leave to the readers.

As the period space is homotopic to $S^{p-1}$, there must be some $p-1$ cycles which generate the $p-1$ homology. It is useful to exhibit such cycles, for this purpose, let us present another picture of the period space.

The period space projects down to $V$. The preimage of an element $x \in V$ is the set of positive $p$ planes which do not lie in $x^{\perp}$, where $x^{\perp}$ denotes the orthogonal complement of $x$ in $V$. Now decompose the vector space $V$ into three parts, $V=V_{+} \cup V_{-} \cup V_{0}$, with

$V_{+}=\{x \in V \mid x \cdot x>0\}, \quad V_{-}=\{x \in V \mid x \cdot x<0\}, \quad V_{0}=\{x \in V \mid x \cdot x=0\}$.

$V_{0}$, usually called the light cone, is diffeomorphic to $\mathbf{R} \times S^{p-1} \times S^{q-1}$. $V_{+}$is diffeomorphic to $\mathbf{R}^{+} \times S^{p-1} \times B^{q}$ and $V_{-}$is diffeomorphic to $\mathbf{R}^{+} \times B^{p} \times S^{q-1}$ and they intersect along $V_{0}$. If $x \in V_{+} \cup V_{0}$, no positive $p$ plane can be perpendicular to it. Therefore, over $V_{+} \cup V_{0}$, the period space has a fiber bundle structure with contractible fibers, $G_{p}^{+}(V)$. If $x \in V_{-}$, the $p$ planes perpendicular to it are the positive $p$ planes which do not lie in $x^{\perp}$ and is itself a sub Self Dual Grassmannian. We denote it by $G_{p}^{+}\left(x^{\perp}\right) . G_{p}^{+}\left(x^{\perp}\right)$ is a submanifold in $G_{p}^{+}(V)$ of codimension $p$. In fact following the same line of the previous argument, one can easily see that $G_{p}^{+}(V)$ is diffeomorphic to an affine space bundle over $G_{p}^{+}\left(x^{\perp}\right)$ of fiber dimension $p$. From here it follows again that the homotopy type of $G_{p}^{+}(V)-G_{p}^{+}\left(x^{\perp}\right)$ is just $S^{p-1}$, a sphere of $p-1$ dimension. Let us denote the complement of $G_{p}^{+}\left(x^{\perp}\right)$ in $G_{p}^{+}(V)$ by 
$\hat{G}_{p}^{+}(V, x)$. Any cycle which generates the $p-1$ homology of $\hat{G}_{p}^{+}(V, x)$ over $x \in V_{-}$is the homology generator of the period space. On the other hand, the $S^{p-1}$ factor in $V_{+}$would also be the generator of the $p-1$ homology of the period space. This simple observation, though elementary, will play a very crucial role in the later development of the whole theory.

Let us summarize the picture when $B$ is a point. The period space of $V=H^{2}(M ; \mathbf{R})$ is homotopic to the sphere $S^{p-1}$ where $p$ is equal to $b^{+}$. The number of $\mathcal{L}$ chambers is greater than one only if $b^{+}=1$. And when $b^{+}=1$, the corresponding period space is homotopic to $S^{0}$ which consists of two points. In fact it is why the usual chamber structure of the ordinary Seiberg Witten theory has a $\mathbf{Z}_{2}$ grading.

For general $B$, the $\mathbf{Z}_{2}$ grading is not enough to parametrize the chambers. In fact we will see shortly it is very typical that the chambers are at least graded by $Z$. Namely there are an infinite number of chambers for even a single $\operatorname{Spin}^{c}$ structure. This would be a general characteristic difference from the ordinary Seiberg-Witten theory.

Suppose we are given a fiber bundle $\mathcal{X}$ over $B$ whose fibers are diffeomorphic to $M$. The cohomology of the fibers $H^{2}\left(\mathcal{X}_{b} ; \mathbf{R}\right)$ form a flat vector bundle $\mathcal{V}$ over the base $B$. We will define the period bundle via $\mathcal{V}$, which generalizes the period space and plays the key role in understanding the chamber structure.

Definition 3.4. The period bundle $\mathcal{P} \mapsto B$ is defined to be the fiber bundle canonically constructed by the flat bundle $\mathcal{V}=H^{2}\left(\mathcal{X}_{b} ; \mathbf{R}\right) \mapsto B$ through the period construction.

The period bundle is a subbundle of $\tilde{\mathcal{P}}=G_{p}(\mathcal{V}) \times_{B} \mathcal{V}$ and has a canonical surjective bundle morphism to $\mathcal{V}$.

From Proposition 3.2, immediately we have

Proposition 3.5. The homotopy type of the period bundle. The period bundle $\mathcal{P}$ is homotopic equivalent to an $S^{p-1}$ bundle over $B$ through fiberwise homotopy.

Let us end this subsection with a remark concerning a result of Kronheimer.

Remark 3.6. Given a four-manifold $M$ with a symplectic form $\omega_{0}$, consider the space

$$
\Lambda_{0}=\left\{\omega \in \Omega^{2}(M) \mid \omega \text { is symplectic and cohomologous to } \omega_{0}\right\} .
$$


For each $\operatorname{Spin}^{c}$ structure detertermined by an element $e \in H^{2}(M ; \mathbf{Z})$ whose index

$$
d=K_{\omega_{0}} \cdot e-e \cdot e
$$

lies in the range $0<d<b^{+}-1$ and which satisfies $\omega_{0} \cdot e<0$, Kronheimer $[\mathrm{K}]$ defined a homomorphism

$$
Q: H_{d-1}\left(\Lambda_{0} ; \mathbf{Z}\right) \longrightarrow \mathbf{Z}
$$

via Seiberg-Witten equations for the $\operatorname{Spin}^{c}$ structure.

From Proposition 3.3, we have

$$
H_{i}(\Gamma-\mathcal{W} A ; \mathbf{Z})=H_{i}(\mathcal{P} ; \mathbf{Z})=H_{i}\left(S^{b^{+}-1} ; \mathbf{Z}\right) .
$$

Tracing Kronheimer's definition of $Q$, it is not hard to see that $Q$ can be extended to any $\operatorname{Spin}^{c}$ structure determined by an element $e \in H^{2}(M ; \mathbf{Z})$ whose index lies in the range $d>b^{+}$and which satisfies $\omega_{0} \cdot e<0$.

This extension is particularly interesting in the case $b^{+}$is equal to one.

\subsection{The chamber structure.}

In this subsection, we will describe the chamber structure in cohomological terms.

Fix a $\operatorname{Spin}^{c}$ structure $\mathcal{L}$ with $L$ as the associated complex line bundle. The map $p r_{\mathcal{L}}$ maps $\Gamma-\mathcal{W} A(\mathcal{L})$ onto sections of $\mathcal{P}$. Furthermore, two pairs in $\Gamma-\mathcal{W} A(\mathcal{L})$ lie in the same $\mathcal{L}$ chamber if and only if their images under $p r_{\mathcal{L}}$ are homotopic as sections of the period bundle $\mathcal{P}$. Therefore we have

Proposition 3.7. Chamber structure. The chambers of a single Spinc structure are classified by fiberwise homotopy classes of sections into $\mathcal{P}$, denoted by $[B, \mathcal{P}]_{f}$.

Unlike the case $b^{+}=1$, the chamber structure can be extremely complicated. For example consider the case $B=S^{a}$ and the fiber bundle $\mathcal{X}$ is a trivial product $M \times B$ with $b^{+}(M)=b$ and $a>b$. Then the set of fiberwise homotopy classes $[B, \mathcal{P}]_{f}$ is given by the set of free homotopy classes from $S^{a}$ to $S^{b},\left[S^{a}, S^{b}\right]$, a very hard object in homotopy theory. In general if we consider a product fiber bundle $\mathcal{X}$, the set $[B, \mathcal{P}]_{f}$ will be given by $\left[B, S^{b^{+}(M)-1}\right]$, the cohomotopy set of $B$, and it does not have a group structure. We do not plan to give a complete description of these chambers. 
Instead, we only like to discuss how to measure the difference of two chambers from cohomological terms. This piece of information will play a crucial role in the derivation of the wall crossing formula.

Whether two sections of $\mathcal{P}$ are fiberwise homotopic to each other is naturally an extension problem: whether the map $f: B \times\{0,1\} \mapsto \mathcal{P}$ given by the two sections can be extended to a map $F: B \times[0,1] \mapsto \mathcal{P}$. This can be achieved via the obstruction theory. The answer to this question is that there will be a sequence of cohomology classes associated to it which measure the obstruction of extension order by order. More precisely, we have $([\mathrm{S}])$

Proposition 3.8. The obstruction of extending the map $f$ to $F$ is measured by a sequence of elements which live in $H^{r}\left(B ; \pi_{r}^{\mathrm{local}}(\mathcal{P})\right)$ for all $r$. Here $\pi_{r}(\mathcal{P})$ is the local system of $r$-th homotopy groups of fibers. In particular if all these obstruction classes vanish, the extension $F$ exists.

As we know that the period space is homotopic to $S^{b^{+}-1}$, the obstruction classes mentioned in the proposition actually lie in $H^{r}\left(B ; \pi_{r}^{\text {local }}\left(S^{b^{+}-1}\right)\right)$ for all $r$. In general the coefficient groups are not the integer group. However there is one element which is particularly important, the principal obstruction. It is the first obstruction for the extension living in $H^{b^{+}-1}(B ; \mathbf{Z})$. Let us denote it by $\operatorname{Obs}\left(s_{1}, s_{2}\right)$ for $\left[s_{1}\right],\left[s_{2}\right] \in[B, \mathcal{P}]_{f}$. Even though the homotopy classes themselves do not have any additive property, the obstruction class discussed here does have the following remarkable property:

$$
\operatorname{Obs}\left(s_{1}, s_{2}\right)+\operatorname{Obs}\left(s_{2}, s_{3}\right)=\operatorname{Obs}\left(s_{1}, s_{3}\right) .
$$

It is this cohomology ciass Obs which will enter the formulation of the wall crossing formula. The subsequent discussions will illustrate this point. Suppose we are given a generic one parameter family of fiber metrics and two forms, parametrized by $I=[0,1]$, over $\mathcal{X} / B$, such that, at 0 and 1 , no reducible solutions occur. Let $\mathcal{S}$ denote the subset in $B \times I$ where reducible solutions occur. The singular locus $\mathcal{S}$ is usually complicated and depends on the one parameter family of metrics and two forms given. But what is important to us is the homology class of the wall crossing locus $\mathcal{S}$ and this is described by

Lemma 3.9. The singular locus $\mathcal{S}$ represents a homology class of $B \times I$ in degree $b^{+}-\operatorname{dim} B-1$. Under the natural identification between the homology of $B$ and the homology of $B \times I$, this class is Poincare dual to the principal obstruction class Obs. 
Proof. Under the map $p r_{\mathcal{L}}$, the one parameter family of fiber metrics and two forms gives a section of $\mathcal{P}^{+}$(precisely, the pull back of $\mathcal{P}^{+}$from $B$ to $B \times I$ ) over $B \times I$. $\mathcal{S}$ is just the singular locus in $B \times I$ where the section lies outside of $\mathcal{P}$ (also the pull back). By transversality we can always perturb the one parameter family such that the family is transverse, so $\mathcal{S}$ is the transveral intersection between the nonzero section and the zero section. such that $\mathcal{S}$ is embedded into $B \times I$ as a submanifold.

As the original extension problem is homotopic in nature, we can assume our period bundle is a $\mathbf{R}^{b^{+}}-\{0\}$ bundle over $B$. To relate the obstruction class with $\mathcal{S}$, we need a CW complex structure on the manifold with boundary $B \times I$ which is transversal to $\mathcal{S}$. One way to obtain such a CW complex structure is to triangulate $B \times I$ such that the triangulation restricted to $\mathcal{S}$ gives a triangulation of $\mathcal{S}$ as a smooth submanifold and take the dual CW complex associated to this particular triangulation. Assume a CW complex structure transversal to $\mathcal{S}$ is given, we want to use it to construct the obstruction class. In this case the map has been defined outside $\mathcal{S}$. Thus over the cells which do not intersect $\mathcal{S}$ at all, there is no obstruction to extend the map from low strata. If we extend the map from the zero stratum to the higher ones, the first time the obstruction appears is in dimension $b^{+}$. The map still extends automatically across any $b^{+}$dimensional cell which is disjoint from $\mathcal{S}$ and those cells are assigned to the zero element in $\pi_{b^{+-1}}\left(S^{b^{+}-1}\right)$. On the other hand, any $b^{+}$cell which intersects $\mathcal{S}$ is assigned to \pm 1 in $\pi_{b^{+}-1}\left(S^{b^{+}-1}\right)$ by the transversality assumption. Whether it is assigned to 1 or -1 is according to the orientation. At the end, we get a $b^{+}$cochain of the CW complex. It is a cocycle on $B \times I$ representing the principal obstruction class. On the other hand, from the Poincare duality, it is exactly the Poincare dual to $\mathcal{S}$. This ends the proof of the lemma.

As just mentioned, this elementary result will be used in the derivation of wall crossing formula later. The readers should keep in mind that it is the cohomology class associated to $\mathcal{S}$, not the manifold $\mathcal{S}$ itself, which is relevant to our later discussion.

Example 3.10 (winding family). Let $M$ be a manifold with $b^{+}>1$ and $\mathcal{X}$ be a trivial bundle with base $S^{b^{+}-1}$. Then there is a canonical chamber characterized by containing the constant parameters, i.e. parameters of constant metrics and constant self dual two forms. With the canonical chamber understood, the chambers are naturally identified with the integer group $\mathbf{Z}$. A family of closed two forms with positive square is called a winding family of two forms if they represent a generator of the $b^{+}-1$ homology of $V^{+}$introduced in ths last subsection, and it is called a symplectic winding family 
if all the two forms are symplectic. It is not hard to see the chamber containing a parameter of constant metric and winding two forms corresponds to \pm 1 under the aforementioned identification.

Interesting symplectic winding familes include hyperkahler families of $\mathrm{K} 3$, the four torus and some $S^{1}$ familes of primary Kodaira surfaces studied in $[\mathrm{Ge}]$.

\section{The wall crossing formula.}

In this section we would like to discuss the wall crossing formula for the family version of Seiberg-Witten invariants. As before let $\mathcal{X} \mapsto B$ be a fiber bundle of four manifolds.

In the first subsection, we would like to discuss the phenomena of wall crossing in some generality. In the second subsection, we would like to specialize to the case of $b^{+}-1=\operatorname{dim} B$ where we can derive a universal wall crossing formula. We call this special case the "critical" case. In the third subsection, we discuss the Fulton-MacPherson spaces.

\subsection{The Kuranishi models.}

In the case $b^{+}-1>\operatorname{dim} B$, we can always move the metrics and two forms perturbation in one parameter family and show by the standard bordism argument that the invariants are actually independent of the metrics and two form perturbations. However in the $b^{+}-1 \leq \operatorname{dim} B$ case as the general position argument could not provide us a completely smooth cobordism between these two moduli spaces. Typically, the parameters (including the metrics and two forms) will hit the singular values somewhere and reducible solutions develop in the bordism. In this case, the bordism is no longer a smooth bordism between these two different moduli spaces. To understand how the invariance property of the "invariants" fails, we need to understand the behavior of the moduli space near the reducible solutions. The following is the fundamental tool to describe the neighborhood of the moduli space near a solution $\left(A_{b}, \psi_{b}\right)$.

Proposition 4.1 (Family Kuranishi Model). Let $\left(A_{b}, \psi_{b}\right)$ be a solution of the family Seiberg-Witten equations at $b$, then a neighborhood of the moduli space near this point $\left(A_{b}, \psi_{b}\right)$ can be described by $F^{-1}(0) / S^{1}$, where

$$
F: T_{b} B \times H^{1}\left(\mathcal{X}_{b} ; \mathbf{R}\right) \times U_{b} \mapsto \operatorname{Ker}\left(d^{*}\right) \times \operatorname{Coker}\left(D_{A_{b}}\right)
$$

is an $S^{1}$ equivariant map and $U_{b}$ is a small ball in $\operatorname{Ker}\left(D_{A_{b}}\right)$. 
The proof of this proposition is standard (see [DK]). When the spinor $\psi_{b}$ is not identically zero on the manifold $\mathcal{X}_{b}$, the $S^{1}$ action is free and the Kuranishi model does not tell us too much other than that it is a smooth point in the moduli space. However when the spinor $\psi_{b}$ is identically zero, $S^{1}$ acts trivially on the point $\left(A_{b}, 0\right)$ and the Kuranishi model describes the local singular behavior near this point. To get useful global topological description for the purpose of calculation, we actually need a global version of the Kuranishi model which generalizes the picture of $b^{+}=1$ case in [LL1]. Let us formulate it as the Global Kuranishi model for the bordism.

Suppose there is a one parameter family of deformation of the parameters $t, 0 \leq t \leq 1$. Let $\mathcal{B}$ denote the bordism connecting the two different moduli spaces at $t=0$ and $t=1$. Suppose that somewhere between 0 and 1 the bordism hits the wall, then we have

Proposition 4.2 (Global Kuranishi Model). Let $\mathcal{S}$ denote the reducible solutions in the bordism $\mathcal{B}$ connecting two different smooth moduli spaces, and we denote the projection map of $\mathcal{S}$ to $I \times B$ by $\pi$ and the image by $S_{0}$. Suppose that $\mathcal{S}$ and $\mathcal{S}_{0}$ are both smooth, then the local neighborhood of $\mathcal{S}$ in $\mathcal{B}$ is described by the following Kuranishi model $F^{-1}(0) / S^{1}$, where

$$
F: T \mathcal{S} \times \pi^{*} N_{I \times B / \mathcal{S}_{0}} \times U \mapsto \operatorname{Ker}\left(d^{*}\right) \times \operatorname{Coker}\left(D_{A}\right)
$$

is again an $S^{1}$ equivariant map, $N_{I \times B / \mathcal{S}_{0}}$ is the normal bundle of $\mathcal{S}_{0}$ inside $I \times B, U$ is a small ball in $\operatorname{Ker}\left(D_{A}\right)$ and $A$ varies along the reducible connections in $\mathcal{S}$.

However this global model still has limited use. Usually we do not know exactly the diffeomorphism type of the singular set $\mathcal{S}$, nor do we know that $\mathcal{S}$ and $\mathcal{S}_{0}$ are smooth. To get a more useful model, it must be replaced by a fatten version.

Let $\mathcal{T}_{B}^{b_{1}}$ be the $T^{b_{1}}$ bundle over $B$ which parametrizes the reducible connections in the family $\mathcal{X} \mapsto B$. It is well known the dimensions of the kernel spaces and cokernel spaces may vary on $\mathcal{T}_{B}^{b_{1}}$ and $\operatorname{Ker}_{B}\left(D_{A}\right)$ and Coker ${ }_{B}\left(D_{A}\right)$ are in general not honest complex vector bundles over $\mathcal{T}_{B}^{b_{1}}$. Tc avoid this difficulty, we can use the technique in [LL1] to perturb the Kuranishi model a little bit (see also the technique developed in $[R]$ ). In the present case, the technique can be applied as the base $\mathcal{T}_{B}^{b_{1}}$ is still compact. And after this slight perturbation, we can always assume $\operatorname{Ker}_{B}\left(D_{A}\right)$ and $\operatorname{Coker}_{B}\left(D_{A}\right)$ are honest vector bundles. As in [LL1], we have

Proposition 4.3 (Modified Global Kuranishi Model). There exists an $S^{1}$ equivariant, fiber preserving map $F$ from a disc subbundle in the 
complex vector bundle $\operatorname{Ker}_{B}\left(D_{A}\right)$ to the complex vector bundle $\operatorname{Coker}_{B}\left(D_{A}\right)$ whose zero set contains the neighborhood of $\mathcal{S}$ in $\mathcal{B}$ as an imbedded subspace.

When $B$ is a single point, the picture reduces to the previous one described in [LL1]. From now on, let us drop $A$ and write $D_{A}$ simply as $D$.

By the modified global modified Kuranishi model, the link space of the singularities Link $(\mathcal{S})$ in $\mathcal{B}$ can be always imbedded inside the projective space bundle formed by $\operatorname{Ker}_{B}(D)$. We do not really care about its topology in detail, in fact we even do not assume that $\mathcal{S}$ and $\mathcal{S}_{0}$ are smooth. What we only need to know is the homology class represented by $\operatorname{Link}(\mathcal{S})$. It is Poincare dual to certain obstruction class over $P\left(\operatorname{Ker}_{B}(D)\right)$. However there is some slight difference than the usual $b^{+}=1$ wall crossing formula. Namely there are two sources of the obstruction classes. One of them is comes from the obstruction bundle which is described in the following definition.

Definition 4.4 ([LL1]). Obstruction Bundle $\mathcal{O} b s$. Let us denote the projection map from $P\left(\operatorname{Ker}_{B}(D)\right)$ to $\mathcal{T}_{B}^{b_{1}}$ by $\pi_{1}$. The obstruction bundle $\mathcal{O} b s$ is given by

$$
\mathcal{O} b s=\pi_{1}^{*} \operatorname{Coker}(D) \otimes H,
$$

where $H$ is the hyperplane line bundle canonically associated with $P\left(\operatorname{Ker}_{B}(D)\right)$.

As argued in Lemma 2.2 in [LL1], $F^{-1}(0)$ can be viewed as the zero set of a section of $\mathcal{O} b s$.

Another source of the obstruction class comes from the period bundle and has been explained in detail in section 3. Suppose that the period maps at 0 and 1 both map into $\mathcal{P}$, then in general there would be some obstruction to extending these into a well defined map from $B \times I$ to the period space. The so-called primary obstruction is measured by a cohomology class $O b s$ which lives in $H^{b^{+}-1}\left(B ; \pi_{b^{+}-1} \mathcal{P}_{b}\right)$. The coefficient is the local system formed by the $\left(b^{+}-1\right)$-th homotopy group of the fiber of the period bundle. As discussed in section 3 , the period space is homotopic to $S^{b^{+}-1}$. Thus the obstruction class $\mathrm{Obs}$ lies in the cohomology with integer coefficient. Then $O b s$ is Poincare dual to the base locus in $B \times I$ where the bordism hits the wall.

Let us state the result for a general invariant $S W(\mathcal{X} / B, \mathcal{L}, \Theta)$. From now on, we will $\operatorname{drop} \mathcal{X} / B$ in the notation and simply write invariant $S W(\mathcal{X} / B, \mathcal{L}, \Theta)$ as $S W(\mathcal{L}, \Theta)$. 
Proposition 4.5. The wall crossing number is calculated by

$$
\pm W C N(\mathcal{L}, \Theta)=\int_{P\left(\operatorname{Ker}_{B}(D)\right)} \Theta \cdot H^{\operatorname{dim}\left(\operatorname{Coker}_{B}(D)\right)} \cdot \text { Obs, }
$$

where the class Obs is pulled back from the base $B$ to $P\left(\operatorname{Ker}_{B}(D)\right)$ and is viewed as a cohomology class living in the projective bundle.

Proof. First,

$$
\pm W C N(\mathcal{L}, \Theta)=\int_{\operatorname{Link}(\mathcal{S})} \Theta .
$$

Following [LL1], we reduce the calculation of the wall crossing number to the calculation on $\mathcal{T}_{B}^{b_{1}}$, with the insertion of the euler class of the obstruction bundle $\mathcal{O} b s$ and the homotopy theoritical obstruction class $O b s$. Since we can assume, as explained in [LL1], that $\operatorname{Coker}_{B}(D)$ is a trivial bundle, we have

$$
\begin{aligned}
\int_{\operatorname{Link}(\mathcal{S})} \Theta & =\int_{P\left(\operatorname{Ker}_{B}(D)\right)} \Theta \cdot \text { Obs } \cdot c_{\operatorname{dim}\left(\operatorname{Coker}_{B}(D)\right)}(\mathcal{O} b s) \\
& =\int_{P\left(\operatorname{Ker}_{B}(D)\right)} \Theta \cdot H^{\operatorname{dim}\left(\operatorname{Coker}_{B}(D)\right)} \cdot \text { Obs. }
\end{aligned}
$$

For the pure invariant, (4.1) takes the following simple form

$$
\int_{P\left(\operatorname{Ker}_{B}(D)\right)} H^{\operatorname{dim} B+b_{1}+\operatorname{dim}\left(\operatorname{Ker}_{B}(D)\right)-b^{+}+1} \cdot O b s .
$$

Notice that whenever the base dimension is lower than $b^{+}-1$ then the obstruction class $O b s$ vanishes automatically and the wall crossing number is zero. This statement coincides with the previous observation that wall crossing phenomena does not exist whenever $\operatorname{dim} B<b^{+}-1$. Another simple fact is if we consider a weight $m$ mixed Seiberg-Witten invariant and $m>\operatorname{dim} B-b^{+}+1$, then the wall crossing number is always zero. This is because if the degree of the cohomology classes from the base will exceed the base $\operatorname{dimension} \operatorname{dim} B$ and the invariants automatically vanish.

In general to calculate the integral (4.1) we need information about the index bundle $I N D_{B}(D)=\operatorname{Ker}_{B}(D)-\operatorname{Coker}_{B}(D) \in K\left(\mathcal{T}_{B}^{b_{1}}\right)$, to be precise, we want to calculate its Chern character $\operatorname{Ch}\left(I N D_{B}(D)\right) \in H^{*}\left(\mathcal{T}_{B}^{b_{1}} ; \mathbf{Q}\right)$. And it is calculated by the Family Index theorem as a push-forward from $\mathcal{X} \times{ }_{B} \mathcal{T}_{B}^{b_{1}}$ to $\mathcal{T}_{B}^{b_{1}}$,

$$
\operatorname{Ch}\left(I N D_{B}(D)\right)=\int_{\mathcal{X} \times{ }_{B} \mathcal{T}_{B}^{b_{1}} / \mathcal{T}_{B}^{b_{1}}} \mathcal{A}_{\mathcal{X} \times{ }_{B} \mathcal{T}_{B}^{b_{1}} / \mathcal{T}_{B}^{b_{1}}} \cdot \operatorname{Ch}(\mathcal{L} \otimes \Omega)
$$


where $\mathcal{A}_{M / N}$ denotes the relative $A$-roof genus of the fiber bundle and $\Omega$ is the tautological line bundle on $\mathcal{X} \times_{f} \mathcal{T}_{B}^{b_{1}}$. When we restrict the line bundle $\Omega$ to each fiber $\mathcal{X}_{b} \times \mathcal{T}_{B}^{b_{1}}$, its first chern class is given by the standard formula $c_{1}\left(\Omega_{b}\right)=\sum x_{i} \cdot y_{i}$, where the $x_{i}, y_{i}$ are some dual basis of $H_{1}\left(\mathcal{X}_{b} ; \mathbf{Z}\right), H^{1}\left(\mathcal{X}_{b} ; \mathbf{Z}\right)$ respectively.

\subsection{The critical case.}

In this subsection, we will concentrate on the critical case where $b^{+}-1=$ $\operatorname{dim} B$. In this case one has an explicit expression of the wall crossing number because of the following lemma.

Lemma 4.6. Suppose $b^{+}-1$ is equal to the dimension of $B$. Then the obstruction class Obs is a top dimensional class in $H^{\operatorname{dim} B}(B ; \mathbf{Z})$.

Obs will produce a number which can be interpreted as the number of times that the parametrized moduli space intersects the wall when the intersections are transverse. And when the intersections are transverse, we can think of $\mathcal{S}_{0}$ as simply a finite number of points.

Let us restrict to the case that the two chambers are consecutive. In this case, $O b s$ reduces $\mathcal{S}_{0}$ to a single point, and the link of the reducible solutions is simply the traditional picture $P(\operatorname{Ker}(D))$ of projective space bundle over $T^{b_{1}}$ in [LL1].

Notice that the wall crossing number between any two consecutive chambers are the same. This simple fact will be crucial to deduce a vanishing result in the next subsection.

Let us first derive the wall crossing formula for the pure invariant. By the above discussion, (4.1) is simply

$$
\int_{P(\operatorname{Ker}(D))} H^{\text {top }}
$$

What we have to calculate is the top power of the hyperplane class on the projective bundle of the index bundle (we know we can choose the representative of $K$-theory class so the $\operatorname{Ker}(D)-\operatorname{Coker}(D)$ is an honest vector bundle). In [LL1], we did some lengthy calculation in the $b^{+}=1$ case. Actually the calculation can be highly simplified by noticing that we calculated the $n$-th Segre class of the Index bundle with $n=b_{1} / 2$. Denote the $n$-th Segre class by $s_{n}$. By the same token, what we want to calculate here is simply $s_{n}(\operatorname{Ind} D)$ with $n=\operatorname{dim} B+b_{1} / 2$. The Segre classes and the Chern classes can be related to each other in a simple way. Namely if one defines 
$S(t)=\sum_{i} s_{i} t^{i}$ and $C(t)=\sum_{i} c_{i} t^{i}$ ( where $c_{i}$ and $s_{i}$ denote the $i$-th chern class and Segre class respectively), then (see $[F]$ )

$$
C(t) S(t)=1
$$

i.e, $S(t)$ is the inverse power series of $C(t)$ ! This observation leads to a simpler computation.

The index calculation is performed on $M \times T^{b_{1}}$. It follows from the family index theorem that the grade $2 i$ term of the expression

$$
\int_{M} A(M) \operatorname{ch}(L) \operatorname{ch}(\Omega)
$$

(where $\Omega \in H^{2}\left(M \times T^{b_{1}} ; \mathbf{Z}\right)$ denotes the first Chern class of the Poincare line bundle) is the $i$-th Chern character, denoted by $c h_{i}$. The first term of the index calculation gives the formal dimension.

The following lemma calculates the Chern character.

Lemma 4.7. $c h_{i}=0$, for all $i>2$ and

$$
\begin{aligned}
& c h_{1}=c_{1}(\mathcal{L}) \cdot \Omega^{2} / 2 ![M] \\
& c h_{2}=\Omega^{4} / 4 ![M] .
\end{aligned}
$$

Proof. Expanding $\operatorname{ch}(\Omega)$ one gets various powers of $\Omega$. From the fact that $\Omega$ is of bi-degree $(1,1)$ on $M \times T^{b_{1}}$, it can not be raised to more than fourth power or it will automatically vanish along $M$.

Notice that it is slightly different from the usual $b^{+}=1$ case where we automatically have the vanishing of $c h_{i}$ with $i \geq 2$ because of the light cone lemma.

Let us temporarily forget about the detailed expression of $c h_{1}$ and $c h_{2}$ in terms of $\Omega$ and $\mathcal{L}$, etc. It makes things simpler to keep the calculation symbolical.

The next step is to prove the following recursive relation between the various $c_{i}$.

Lemma 4.8. The chern classes of the index bundle satisfy the recursive formula

$$
c_{i}=\frac{1}{i}\left(c_{i-1} c h_{1}-2 c_{i-2} c h_{2}\right) \text {. }
$$


Proof. Let us look at the defining equation of the cohomology of the projective bundle (the splitting space).

$$
x^{m}-c_{1} x^{m-1}+c_{2} x^{m-2} \ldots \ldots \ldots+(-1)^{m} c_{m}=0,
$$

where $m$ is the complex dimension of the bundle. Notice that the variable $x$ can represent any of the Chern roots of the bundle $\lambda_{i}, i=1, \ldots, m$. Thus we can plug all the Chern roots in to get $m$ equations. Summing these $m$ equations we get

$$
\left(\sum_{i} \lambda_{i}^{m}\right)-c_{1}\left(\sum_{i} \lambda_{i}^{m-1}\right) \ldots . .+(-1)^{m} m c_{m}=0 .
$$

Using the fact $c h_{i}=0, i>2$ and $c h_{1}=\sum_{i} \lambda_{i}, 2 c h_{2}=\sum_{i} \lambda_{i}^{2}$ we see that

$$
m c_{m}=c_{m-1} c h_{1}-2 c_{m-2} c h_{2} .
$$

This proves the recursive formula for $i=m$. For general $i$, one divides the equation by $x^{k}$ and get

$$
x^{m-k}-c_{1} x^{m-1-k} \ldots \ldots . c_{m} x^{-k}=0 .
$$

Plugging in the Chern roots and summing over $i$ we get an equation which can be reduced to the following form after using $c h_{i}=0, i>2$.

$$
\begin{aligned}
& \sum_{l, l<k}(-1)^{l-k}\left(c_{m-l}\left(\sum_{i} \lambda_{i}^{l-k}\right)\right) \\
&+m c_{m-k}-c_{m-k-1} c h_{1}+2 c_{m-k-2} c h_{2}=0 .
\end{aligned}
$$

To show that the recursive formula is true, one only needs to show that the first sum over $l$ is equal to $-k c_{m-k}$.

To show this let us play a trick. Denote $\sum_{p>0} \sum_{i}\left(\lambda_{i} t\right)^{-p}$ by $F(t)$, the Laruent series in $t$. Then the sum in (4.3) can be expressed as the $m-k$ power coefficient in $t$ of $C(t) \cdot F(t)$. On the other hand, if we commute the sum over $p$ and $i$ in the definition of $F(t)$ and extend $\mathrm{p}$ to $\geq 0$, then we find that $F(t)$ can be re-written as

$$
\begin{aligned}
\sum_{i}\left(\sum_{p \geq 0}\left(\lambda_{i} t\right)^{-p}-1\right) & =\sum_{i}\left(\left(1+\left(\lambda_{i} t\right)^{-1}\right)^{-1}-1\right) \\
& =-\sum_{i}\left(\left(1+\lambda_{i} t\right)^{-1}\right)
\end{aligned}
$$


Thus $C(t) F(t)$ can be simplified as

$$
-\prod_{i}\left(1+\lambda_{i} t\right)\left(\sum_{i}\left(1+\lambda_{i} t\right)^{-1}\right)=-\sum_{i}\left(\prod_{j \neq i}\left(1+\lambda_{j} t\right)\right) .
$$

The final expression is related to taking derivative on a product of linear factors. In fact let us call $G(s)=s^{m} C\left(s^{-1}\right)=\sum_{r} c_{r} s^{m-r}$. Then $-s^{1-m} d /\left.d s(G(s))\right|_{s=t^{-1}}$ is exactly equal to this expression. Thus it is easy to see that the $(m-k)$-th order term in $t$ is exactly $-k c_{m-k}$. This proves the lemma.

Knowing the recursive relation, it follows that one can derive an O.D.E. for $C(t)$. equation of $C(t)$. More precisely, view $C(t)$ as an element in $R\left[\left[\lambda_{1}, \lambda_{2}, \ldots, \lambda_{m}, t\right]\right]$, then we have

Lemma 4.9. The series $C(t)$ formally satisfies the following differential equation,

$$
\frac{d}{d t} C(t)=\left(c_{1}-2 c h_{2} t\right) C(t)
$$

On the other hand we know that $C(0)=1$. With this initial condition of the differential equation, we immediately get

$$
C(t)=\operatorname{Exp}\left(c h_{1} t-c h_{2} t^{2}\right) .
$$

Therefore, by (4.2),

$$
S(t)=\operatorname{Exp}\left(-c h_{1} t+c h_{2} t^{2}\right) .
$$

To get the $n$-th order term of $S(t)$ we collect the terms on the right hand side of (4.4) which is of the type $t^{i} t^{2 j}$ such that $i+2 j=n$. Each term of this type contributes $(-1)^{i} c h_{1}^{i} c h_{2}^{j} / i ! j$ !, thus we get our final formula.

Theorem 4.10. The wall crossing number of the pure invariant in the critical case is expressed in term of the Chern characters as follows,

$$
\pm W C N(\mathcal{L}, H)=\sum_{i+2 j=b_{1} / 2}(-1)^{i} c h_{1}^{i} \cdot c h_{2}^{j} / i ! j !\left[T^{b_{1}}\right] .
$$

This theorem applies to section invariants as well and we state it as a proposition.

Proposition 4.11. The wall crossing number of section invariants is also given by (4.5). 
Proof. Though different sections $s$ give rise to different cohomology classes $e(s)$ on the moduli space which is a fiber space over $B$, they all determine the same cohomology class on $P(\operatorname{Ker}(D))$, and this class is nothing but the hyperplane class $H$. Hence they all have the same wall crossing number as $W C N(\mathcal{L}, H)$.

If one uses the fact that $c h_{2}=0$ for $b^{+}=1$ four-manifolds, one immediately recovers the wall crossing formula in [LL1]. The explicit formula of $c h_{i}$ depends on some detailed structure of the cohomology ring structure of $M$. However, when $M$ has $b_{1}=0$, the wall crossing number of the pure invariant is simply given by \pm 1 . A particular interesting example is an $S^{2}$ winding family of K3 surfaces (remember a K3 surface has $b^{+}=3$ ).

For any manifold with $b_{1} \neq 0$, we would like to describe the form of its wall crossing formula in term of its cohomology ring pairing. First we know that $c h_{1}$ is calculated by $c_{1}(\mathcal{L}) \cdot \Omega^{2} / 2 ![M] \in H^{2}\left(T^{b_{1}}\right)$ and $c h_{2}$ is calculated by $\Omega^{4} / 4 ![M] \in H^{4}\left(T^{b_{1}}\right)$. Let us define $\left(\begin{array}{c}b_{1} \\ 2\end{array}\right)$ number of linear functional $q_{i j}$ on $H^{2}(M)$ by cupping $c_{1}(\mathcal{L})$ with $x_{i} \cdot x_{j}$. Then $c h_{1}$ can be re-expressed as $\sum_{i<j} q_{i j}(\mathcal{L}) y_{i} \cdot y_{j} \in H^{2}\left(T^{b_{1}}\right)$. Likewise we can introduce $\left(\begin{array}{c}b_{1} \\ 4\end{array}\right)$ numbers, denoted by $\epsilon_{i j k l}$ by cupping $x_{i}, x_{j}, x_{k}, x_{l}$ together. Then $c h_{2}$ can be expressed as $\sum_{i<j<k<l} \epsilon_{i j k l} y_{i} \cdot y_{j} \cdot y_{k} \cdot y_{l} \in H^{4}\left(T^{b_{1}}\right)$.

Even though the formula looks complicated we are still able to conclude that the wall crossing formula is a polynomial function in $c_{1}(\mathcal{L})$ of degree at most $b_{1} / 2$. Whether this function is totally trivial or is honestly a polynomial of degree $b_{1} / 2$ really depends on the ring structure of the cohomology of $M$. In case we know that the cup product of $H^{1}$ is nondegenerate, we always know that the wall crossing number behaves honestly as a $b_{1} / 2$ degree polynomial. This is the case for Kahler surfaces where the hard Lefschetz theorem implies the non-degeneracy of the cup product. On the other hand if the cup product on $H^{1}$ is totally null, it automatically implies the vanishing of wall crossing number of the section invariants of all the Spin ${ }^{c}$ structures.

Let us look at an important example, any $S^{2}$ family of the four torus $T^{4}$ $\left(T^{4}\right.$ also has $\left.b^{+}=3\right)$. Unlike the case of the $\mathrm{K} 3$ surface, the wall crossing numbers of $T^{4}$ are no longer just 1 or -1 and they are expressed as a degree two polynomial in $\mathcal{L}$, as was expected from the Kahler property of $T^{4}$. A simple calculation tells us that $c h_{1}=\left(c_{1}(\mathcal{L}) / 2\right)^{2}$ and $c h_{2}=1$. Since $W C N(\mathcal{L}, H)=c h_{1}^{2} / 2+c h_{2}$, we have

Corollary 4.12. The wall crossing number of the pure invariant of a $\operatorname{Spin}^{c}$ structure $\mathcal{L}$ on a $S^{2}$ family of $T^{4}$ are given by

$$
\pm W C N(\mathcal{L}, H)= \pm\left(\frac{1}{8} c_{1}(\mathcal{L})^{2}+1\right) .
$$


Before going on to discuss the wall crossing formula for general SeibergWitten invariants in the critical case, we want to make a remark concerning the noncritical case.

In the critical case, the formula is very neat because $c h_{i}=0, i>2$. However, even without this assumption, $c_{i}$ still satisfies the following recursive relation,

$$
c_{i}=\frac{1}{i}\left(c_{i-1} c h_{1}-2 c_{i-2} c h_{2}+3 ! c_{i-3} c h_{3}+\cdots\right),
$$

as can be derived along the line of lemma 4.8.

Similiarly $C(t)$ satisfies the following differential equation,

$$
\frac{d}{d t}(C(t))=C(t)\left(c h_{1}-2 ! c h_{2}+3 ! c h_{3}-4 ! c h_{4}+\cdots\right) .
$$

And the same initial condition leads to the solution

$$
\begin{aligned}
C(t) & =\operatorname{Exp}\left(c h_{1} t-c h_{2} t^{2}+2 ! c h_{3} t^{3}-\cdots\right) \\
& =\operatorname{Exp}\left(\sum(-1)^{i-1} \operatorname{ch}_{i} t^{i}(i-1) !\right) .
\end{aligned}
$$

The derivation is done along the same line and we skip the proof. However the final expression of the Segre classes is much more lengthy than before. This formula has certain application if we consider family wall crossing formula of the noncritical cases.

Next let us discuss the invariants induced from $H_{1}(M ; \mathbf{Z})$. In this situation, we must insert certain power of the euler class and certain power of the $\mu$ map images to the projective space bundle over $T^{b_{1}}$. We denote the class on $P(\operatorname{Ker}(D))$ induced by $\mu(\zeta)$ also by $\mu(\zeta)$. It is well known that this class further determines a class on the torus $T^{b_{1}}$. By further abusing notation, we denote this class by $\mu(\zeta)$ as well. As before, the effect of introducing the new classes merely reduces the effective dimension of the torus $T^{b_{1}}$.

Theorem 4.13. Given $\zeta_{1}, \ldots, \zeta_{q} \in H_{1}$, the wall crossing number of $S W\left(\zeta_{1}, \ldots, \zeta_{q}\right)$ is given by

$$
\begin{aligned}
& \pm W C N\left(\zeta_{1}, \ldots, \zeta_{q}\right) \\
& =\sum_{i+2 j=\frac{b_{1}-q}{2}}(-1)^{i}(i+j, i) c h_{1}^{i} \cdot c h_{2}^{j} /(i+j) ! \mu\left(\zeta_{1}\right) \cdots \mu\left(\zeta_{q}\right)\left[T^{b_{1}}\right] .
\end{aligned}
$$

In general the wall crossing number will become a polynomial of $c_{1}(\mathcal{L})$ of degree less than or equal to $b_{1} / 2-q$. We would like to illustrate this by studying the primary Kodaira surfaces. 
The primary Kodaira surfaces are the complex surfaces (non-Kahler) which are quotients of $\mathbf{R}^{4}$ with $b^{+}=2$ and $b_{1}=3$. Let $(x, y, z, t)$ be the coordinate of $\mathbf{R}^{4}$, the discrete group is generated by $(x, y, z, t+1),(x, y, z+$ $1, t),(x, y+1, z, t)$ and $(x+1, y, z+\lambda y, t)$ where $\lambda$ is a fixed positive number. (We exclude $\lambda=0$ case as it gives $T^{4}$.) In symplectic geometry they are known to be constructed by Thurston as first examples of symplectic manifolds which are not Kahler.

If we project $M$ to $(x, t),(y, t)$ or to $(x, y)$, we obtain three descriptions of the primary Kodaira surfaces as $T^{2}$ bundle over $T^{2}$ with $d x \wedge d t, d y \wedge d t$ and $d x \wedge d y$ as Poincare duals to the fibres.

It is easy to see that $H^{1}(M ; \mathbf{R})$ is generated by three differential forms $d x, d y, d t$. On the other hand, $H^{2}(M ; \mathbf{R})$ is generated by $d x \wedge d t, d y \wedge$ $d t, d y \wedge d z, d x \wedge(d z-\lambda x d y)$. From here it is easy to see that the subspace $d x \wedge d t, d y \wedge d t$ is the two dimensional subspace of $H^{2}$ generated by $H^{1}$. Comparing with $T^{4}$, one difference is that the ring structure on $H^{1}$ is not nondegenerate. There are two nice $S^{1}$ symplectic winding familes,

$$
\begin{aligned}
& \omega_{0}(\theta)=\cos \theta d x \wedge d t+\sin \theta d y \wedge d z \\
& \omega_{1}(\theta)=\cos \theta d y \wedge d t+\sin \theta d x \wedge(d z-\lambda x d y) .
\end{aligned}
$$

And for generic $\theta$, the first fibration is symplectic with respect to $\omega_{0}(\theta)$, the second is symplectic with respect to $\omega_{1}(\theta)$.

The critical dimension is $b^{+}-1=1$ now, thus it is natural to consider the family version of SW invariants with $\mathcal{X}=M \times S^{1}$. As the base is odd dimensional, it is easy to deduce that the wall crossing numbers are all zero for the pure invariant! Thus we must consider invariants involving $H_{1}$. The same observation also tells us that the insertion of an even number of classes in $H_{1}$ always give the answer zero. On the other hand, if we insert more than $b_{1}$ number of classes, it is also zero. Therefore, we will only look at the cases $q=1$ and $q=3$.

If we insert three classes into the definition of the invariants, it can be easily seen that the wall crossing numbers are given simply by

$$
\pm \mu\left(\zeta_{1}\right) \mu\left(\zeta_{2}\right) \mu\left(\zeta_{3}\right)\left[T^{3}\right] .
$$

Whether it is zero or not depends only on whether $\zeta_{i}$ are linear independent in $H_{1}(M, \mathbf{R})$ or not. However the wall crossing formula can be applied only if the $\operatorname{Spin}^{c}$ structure has a moduli space whose dimension is at least three.

On the other hand, the $q=1$ case is less limited, the moduli spaces are only required to have dimensions bigger than zero. 
Any $\operatorname{Spin}^{c}$ structure can be represented as a cohomology class $m_{1} d x \wedge$ $d t+m_{2} d y \wedge d t+m_{3} d y \wedge d z+m_{4} d x \wedge(d z-\lambda x d y)$ with $m_{i} \equiv 0(\bmod 2)(\mathrm{We}$ have used the fact that these differential forms are actually an integral basis of $H^{2}$ ). In the wall crossing formula, $c h_{2} \equiv 0$ as the cup product pairing on $H^{1}$ is degenerate. Thus the wall crossing formula is given by

$$
W C N(\mathcal{L}, \zeta)=-c_{1}(\mathcal{L} / 2) \cdot \Omega^{2} / 2 ![M] \cdot \mu(\zeta)\left[T^{3}\right] .
$$

Denoting $\zeta^{*}$ by $n_{1} d t+n_{2} d y+n_{3} d x$, we find a numerical expression

$$
W C N(\mathcal{L}, \zeta)=\frac{-\left(m_{3} \cdot n_{2}+m_{4} \cdot n_{3}\right)}{2} .
$$

Finally, to see the structure of the invariants, we can form the generating series of them. Let us define the generating series $W C N(\zeta)$ to be

$$
\begin{aligned}
\sum_{k_{1}, k_{2}, k_{3}, k_{4}} W C N\left(2 \left(k_{1} d x \wedge d t+k_{2} d y\right.\right. & \wedge d t+k_{3} d y \wedge d z \\
& \left.\left.+k_{4} d x \wedge(d z-\lambda d y)\right), \zeta\right) t_{1}^{k_{1}} t_{2}^{k_{2}} t_{3}^{k_{3}} t_{4}^{k_{4}}
\end{aligned}
$$

With some simple reduction we get

$$
W C N(\zeta)=\frac{-\left(n_{2} t_{3}+n_{3} t_{4}-\left(n_{2}+n_{3}\right) t_{3} t_{4}\right)}{\left(1-t_{1}\right)\left(1-t_{2}\right)\left(1-t_{3}\right)^{2}\left(1-t_{4}\right)^{2}}
$$

Similiarly we define $\operatorname{WCN}\left(\zeta_{1}, \zeta_{2}, \zeta_{3}\right)$ and it can be easily computed as

$$
\prod_{i=1}^{4} \frac{1}{\left(1-t_{i}\right)} \phi\left(\zeta_{1}^{*} \wedge \zeta_{2}^{*} \wedge \zeta_{3}^{*}\right)
$$

where $\phi: \wedge^{3} H^{1} \mapsto R$ maps $d x \wedge d y \wedge d t$ to 1 .

It is easy to deduce that if $\mathcal{L}$ corresponds to a cohomology class not of the form $m_{1} d x \wedge d t+m_{2} d y \wedge d t$, then the Seiberg-Witten moduli space in the first winding chamber is nonempty if $\operatorname{dim} \mathcal{M}_{\mathcal{L}} \geq 1$.

\subsection{Important examples of the non-critical case: Fulton-MacPherson spaces.}

In the last subsection, we are able to derive the family wall crossing formula in the critical case. In that case, the formula is universal in the sense that it is independent of the details regarding the topology of the fiber bundle. When $\operatorname{dim} B$ is bigger than $b^{+}-1$, the picture is much more complicated. In this 
subsection, we will thoroughly investigate an important class of examples, the Fulton-MacPherson spaces.

The discussion of the Fulton-MacPherson spaces has a twofold purpose. On one hand, it demonstrates the complexity of the problem that it is usually difficult to derive an explicit formula in the non-critical case. In general, the answer and the detailed calculations depend on the topology of the given fiber bundle. As a result, we are not able to get an universal formula as we did before.

On the other hand, it was suggested to the authors by G. Tian that nodal Gromov-Witten invariants should be differentiable invariants and this might be proved using family Seiberg-Witten invariants over some natural families. We believe the Fulton-MacPherson families are the right famlies.

The Fulton-MacPherson spaces will be used to build up a fiber bundle. If we want to apply this scheme to K3, $T^{4}$ or the primary Kodaira surfaces, we will thicken the base by $S^{2}$ or $S^{1}$. In general, we will thicken the base by $S^{b^{+}-1}$. This family is ultimately related to the counting of nodal (or higher singularities) pseudo-holomorphic curves. In fact after certain transformation, the wall crossing formula in these particular examples can be shown to be closely related to the enumeration problem in algebraic geometry. This also strongly supports (as an nontrivial example) the general conjecture between the family Seiberg-Witten theory and the family Gromov theory. In fact, the result will be compared with the results from the blow up formula by A.K. Liu in another up-coming paper. The fact that they coincide gives rise to a nontrivial consistency check of the calculations.

First, let us introduce the concept of the Fulton-Macpherson space. Even though their construction is completely general, we will restrict our attention to almost complex four manifolds only. Let $Y$ be an almost complex manifold. Let $Z$ be an almost complex submanifold inside $Y$ of codimension $m$. Then there is a well defined process of blowing up the submanifold $Z$ inside $Y$. Topologically, it is given by replacing an almost complex neighborhood of $Z$ by a projective bundle over $Z$ such that the new manifold has a canonical almost complex structure. If $Y$ is an almost complex manifold with a symplectic structure, one can perform the symplectic blow up by gluing the symplectic structure on the complement of the neighborhood and the symplectic structure on the projective space bundle by some cut off process. For the details of the construction, please consult Guillemin- Sternberg and McDuff's papers [GS] and [M2]. From now on $M$ will be assumed to be an almost complex four-manifold.

A set of ordered $n$ distinct points on $M$ is equivalent to a point in the configuration space $M^{n}(\triangle)=\left(M^{n}\right.$-diagonals $)$, where $M^{n}$ is the product of 
$n$ copies of $M$. The natural question to ask is whether $M^{n}(\triangle)$ has a smooth compactification which respects the action of the symmetric group $S_{n}$. It was Fulton and MacPherson ([FM]) who first explicitly constructed a good compactification of the configuration space when $M$ is a smooth complex manifold. Later a similiar construction was also used by Kontsevich and other people to study Chern Simons theory.

The Fulton-MacPherson space $M[n]$ is a nice compactification of $M^{n}(\triangle)$ such that the compactifying divisors have certain moduli meaning. Moreover, the space $M[n]$ is smooth, $S_{n}$ equivariant and maps surjectively to the space $M^{n} . M[n]$ can be described in several ways, we will construct $M[n]$ by a sequence of $2^{n}-n-1$ blowups from $M^{n}$. For each of the $2^{n}-n-1$ subset $S$ of $\{1, \ldots, n\}$ with cardinality at least two, let $\Delta_{S} \subset M^{n}$ be the diagonal where the points $x_{i}$, for $i$ in $S$, coincide. We start with $M[2]$, it is just the blowup of $M \times M$ along the diagonal which corresponds to the diagonal $\Delta_{\{1,2\}}$. Suppose $M[n]$ is constructed with a map to $M^{n}$. We will construct $M[n+1]$ by blowing up all diagonals $\Delta_{S}$ in $M^{n+1}$ (or more precisely, the preimage of the diagonals under the map $M[n] \times M \mapsto M^{n+1}$ ) where $n+1$ is an element of $S$. The blowups are done in $n$ steps. The first step is to blow up the diagonal $\Delta_{\{1, \ldots, n\}}$, next we blow up the proper transform of the $n-1$ diagonals $\Delta_{S}$ where $S$ has cardinality $n$. The $k$-th step is to blow up the proper transform of the diagonals $\Delta_{S}$ where $S$ has cardinality $n+2-k$. Notice that after the $k$-th step, the proper transform of the diagonals to be blwon up in the $k+1$-th step become disjoint and the order of teh blow up of these diagonals in this step is irrelavant. This is exactly why $M[n]$ preserves the symmetry of the group $S_{n}$. It is not hard to see that all the blowups are along submanifolds of complex codimension 2 or 3 .

Given this inductive description of $M[n]$, one may wonder whether $M[n+$ 1] $\mapsto M[n]$ gives rise to a smooth fiber bundle which parametrizes blowups. Unfortunately some fibers of this map are singular. When a point lies in $M^{n}(\Delta)$, the fibers are all diffeomorphic to $M$ with these $n$ points blown up. On the other hand when two or more points collide, the fibers themself will become unions of several normal crossing four manifolds. One of them is $M$ with several points blown up, the other components are all rational (i.e. $C P^{2}$ with fewer points blown up). They intersect each other in $C P^{1}$ and the total number of exceptional $C P^{1}$ sums up to be $n$. As the current Seiberg-Witten theory is developed for the smooth fiber bundles, we restrict our discussion to this case. (The Seiberg-Witten theory for singular spaces or singular fibrations is an interesting subject of its own.)

However a simple modification will give us the desired bundle. We start from the space $M[n] \times M$ and denote it by $\mathcal{X}_{0}$. The composi- 
tion of $M[n] \mapsto M^{n}$ with the $i$-th $(1 \leq i \leq n)$ projection map gives rise to $n$ graph $V_{i}: M[n] \mapsto M[n] \times M$, corresponding to the diagonals $\Delta_{\{1, \ldots, n+1\}}, \ldots, \Delta_{\{n, \ldots, n+1\}}$. Instead of first blowing up some codimension 3 diagonals corresponding to subsets with more than two elements to get a space, denoted by $M[n]^{+}$in [FM], and then blow up the proper transform of $V_{i}$ to get $M[n+1]$, we only blow up $M[n] \times M$ successively along the graphs $V_{i}$. More precisely, we first blow up the first graph $V_{1}$ and call the new space $\mathcal{X}_{1}$. Next we blow up the proper transformation of the second graph $V_{2}$ and call the new space $\mathcal{X}_{2}$. After $n$ steps, we get the space $\tilde{M}[n]=\mathcal{X}_{n}$.

As we are always blowing up smooth manifolds along smooth centers, the exceptional divisors will be some projective $P^{1}$ bundles. On the other hand, as the graphs intersect each other, one blow up will definitely affect the topological types of the other divisors we have already blown up. Thus we do not expect the final $n$ smooth exceptional divisors to be simply projective $P^{1}$ bundles. It rather looks like a blown up manifold which comes from the $P^{1}$ bundle by blowing up certain low dimensional locus. Nevertheless, we still have the following important proposition.

Proposition 4.14. The smooth almost complex manifold $\tilde{M}[n]$ forms a smooth fiber bundle over $M[n]$ whose fibers are almost complex fourmanifolds all diffeomorphic to the manifold $M \# n \overline{C P}^{2}$.

Proof. We need to show the natural map $\tilde{M}[n] \mapsto M[n]$ has surjective differential. Away from the exceptional divisors, this is clear. On the other hand, the exceptional divisors map to the graphs $V_{i}$ with surjective differentials, and since $V_{i}$ are graphs the differentials of $V_{i} \mapsto M[n]$ are isomorphisms. Thus $\tilde{M}[n] \mapsto M[n]$ is a fiber bundle. The fibers are diffeomorphic to $M \# \overline{C P}^{2}$ because the fibers over $M^{n}(\Delta)$ are just $M$ blown up at $n$ distinct points.

The space $\tilde{M}[n]$ does not allow an $S_{n}$ action, the $S_{n}$ symmetry has been broken when we mark the graphs and determine the order of blowing up using these markings. Also notice that the fibers actually inherit the almost complex structures from the blow up process. Thus our fiber bundle $\tilde{M}[n]$ is a universal fiber bundle parametrizing all the inherited almost complex structures on the manifold $M$ with $n$ points blown up.

If $M$ is chosen to be a symplectic four-manifold, then we can choose the almost complex structure on $M$ to be compatible with the given symplectic form. If so, the manifold $M^{n}$ becomes a symplectic manifold, too. By performing the symplectic blowing up, $\tilde{M}[n]$ also carries a (non-canonical) 
symplectic structure such that the fibers of the fibration $\tilde{M}[n] \mapsto M[n]$ are symplectic. If one considers the hyperwinding family of symplectic forms, one can also construct a family of almost complex structures compatible with the given family of symplectic forms and that the canonically defined sections in these fiber bundles $\mathcal{X}_{n}$ are simutaneously almost complex(and symplectic) with respect to the family of almost complex structures. Thus a family version of the Guillemin-Sternberg-Mcduff construction gives us a family of symplectic structures on the total space $\tilde{M}[n]$ such that the fibers are symplectic with respect to a family of symplectic forms closely related to the original hyperwinding family.

From now on, we will choose $\tilde{M}[n] \times S^{b^{+}-1}$ as the total space $\mathcal{X}$ and $M[n] \times S^{b^{+}-1}$ as the base $B$ and discuss the Seiberg-Witten theory on the bundle $\mathcal{X} \mapsto M[n] \times S^{b^{+}-1}$. As the fiber bundles are not of critical dimensions, as mentioned in $\S 2$, to define the Seiberg-Witten invariants, we need to perturb the Seiberg-Witten equations by the large self-dual two forms to avoid reducible solutions.

In the derivation of the wall crossing formula, we do not require the manifold $M$ to be symplectic. However the later application will be for this case. Thus we are free to use the symplectic forms to perturb the SeibergWitten equations. If $M$ is symplectic with $b^{+}=1$, there are two ways of perturbing the equations. Given a symplectic two form, we can perturb the equations either by $r \omega$ or $-r \omega$. Letting $r \mapsto \infty$, there are two chambers and two different invariants. Therefore one should study the family wall crossing formula to understand how the invariants jump. When $b^{+}$is greater than one, we can consider different winding chambers of self dual two forms. The family wall crossing formula also makes sense in these cases.

As the fibers are blown up from manifolds diffeomorphic to $M$, naturally we are interested in the $\operatorname{Spin}^{c}$ structures which have the following schematic form,

$$
\mathcal{L}=\mathcal{L}_{0}-\sum_{i}\left(2 m_{i}+1\right) E_{i}
$$

where $\mathcal{L}_{0}$ is a $\operatorname{Spin}^{c}$ structure on $M, E_{i}$ is the line bundle which is Poincare dual to the homology class representing the $i$-th exceptional class and each $m_{i}$ is an integer. Notice that these line bundles $E_{i}$ give the exceptional line bundles of each fiber when they are restricted to each individual fiber. The geometric meaning of these numbers $m_{i}$ will be clear in the later discussion. As before, we still require the dimension of its moduli space to be non- 
negative, i.e.,

$$
\begin{aligned}
& 1 / 4\left(\mathcal{L}_{0}^{2}-\sum_{i}\left(2 m_{i}+1\right)^{2}-(2 \tilde{\chi}+3 \tilde{\sigma})\right)+4 n+\left(b^{+}-1\right) \\
& =1 / 4\left(\mathcal{L}_{0}^{2}-\sum_{i}\left(2 m_{i}+1\right)^{2}-(2 \chi+3 \sigma)+n\right)+4 n+\left(b^{+}-1\right) \\
& \geq 0 .
\end{aligned}
$$

Let us analyze the chamber structure of this family. By Proposition 3.5 , the period bundle is homotopic to an $S^{b^{+}-1}$ bundle over $M[n] \times S^{b^{+}-1}$. Thus by Proposition 3.6, the chambers are classified by the set of fiberwise homotopy classes of the sections $\left[M[n] \times S^{b^{+}-1}, \mathcal{P}\right]_{f}$. Usually this set is complicated. We are only interested in a subset consisting of those homoptopy classes of sections whose restriction from $M[n] \times S^{b^{+}-1}$ to $M[n] \times\{p t\}$ is nullhomotopic. This subset can be identified with $\mathbf{Z}_{2}$ if $b^{+}=1$ and $\mathbf{Z}$ if $b^{+}>1$. As the group $Z$ is an infinite cyclic group generated by 1 , we can calculate the wall crossing number from the zero winding chamber to the first winding chamber. Notice that in this case the obstruction class $O b s$ is the pull back $M[n] \times S^{b^{+}-1} \longrightarrow S^{b^{+}-1}$ of the generator of $H^{b^{+}-1}\left(S^{b^{+}-1} ; \mathbf{Z}\right)$. By inserting this special obstruction class into the wall crossing formula we conclude that the calculation can be performed on certain projective space bundle over the torus fibration with $M[n]$ as its base. Notice that it is not necessarily true that the singular locus inside $M[n] \times S^{b^{+}-1}$ is diffeomorphic to $M[n]$. However, we will conveniently adopt this picture, as the wall crossing formula is purely homological.

In this special case, the wall crossing number calculation is again reduced to the calculation of the top power of the hyperplane class over the torus fibration (when $b_{1}(M)=0$, the torus fibration reduces to $M[n]$ itself). As argued in the critical case, this calculation is exactly equivalent to the calculation of the Segre classes of the Index bundle. It is a very complicated calculation if we want to directly apply the family index theorem. Though there is a slight modification which simplifies the calculation to certain degree, we are not able to calculate the final result explicitly. What we can do is to reduce the calculation to a problem in algebraic geometry. In the process, it would be manifest how the family version of Seiberg-Witten invariants correspond to the similar story on the Gromov side -in terms of algebraic geometry.

We change the strategy as follows. Instead of making $\operatorname{Coker}_{M[n]}(D)$ a trivial bundle by adding its $K$ theory inverse, we do it to $\operatorname{Ker}_{M[n]}(D)$. Thus 
we can assume that there is some representative of the Index bundle such that $\operatorname{Ker}_{M[n]}(D)$ is trivial, while $\operatorname{Coker}_{M[n]}(D)$ absorbs all the topology of the bundles. Since Coker ${ }_{M[n]}(D)$ forms the inverse bundle of $I N D_{M[n]}(D)$ in the $K$ group, the Segre classes of $I N D_{M[n]}(D)$ are exactly the Chern classes of $\operatorname{Coker}_{M[n]}(D)$. In other words, if we want to calculate the top power of the hyperplane bundle, it is equivalent to calculate the top dimensional (in term of the base manifold) Chern class of the Cokernel bundle. We would like to demonstrate that there is some special candidate for the $\operatorname{Coker}_{M[n]}(D)$ which allows us to interpret the problem in term of algebraic geometry.

Before going into the detail, let us explain the relationship between the wall crossing numbers and the family version Seiberg-Witten invariants. In general to apply the wall crossing formula to calculate the Seiberg-Witten invariants, we need the information of one specific chamber. This is exactly the case one can achieve for the ruled and rational surfaces $\left(b^{+}=1, B=p t\right)$ and hyperwinding families of the K3 surfaces and $T^{4}$ where we have some sort of vanishing theorem for the invariants due to the existence of special metrics of nonnegative scalar curvature. Then the wall crossing number is exactly the Seiberg-Witten invariants. In our case the same conclusion still holds, but we offer a different argument. Instead, we would like to prove that the pure Seiberg-Witten invariants vanish in the unwinding chamber.

\section{Proposition 4.15 (Vanishing result for Seiberg-Witten Invariants).} The pure family Seiberg-Witten invariants of $b^{+}>1$ family $\tilde{M}[n]$ vanish in the zero winding chamber.

Proof. If $b^{+}$is even, the pure invariants of these families are automatically zero by dimension reason. Thus we only need to study the case when $b^{+}$is odd. Denote the pure Seiberg-Witten invariant in the $\mathrm{i}$-th winding chamber by $S W(\mathcal{L}, H, i)$, then

$$
\begin{aligned}
W C N(\mathcal{L}, H) & =S W(\mathcal{L}, H, 1)-S W(\mathcal{L}, H, 0) \\
& =S W(\mathcal{L}, H, 0)-S W(\mathcal{L}, H,-1) .
\end{aligned}
$$

On the other hand, we can use an orientation reversing diffeomorphism of $S^{b^{+}-1}$ to map one chamber into another. We conclude that

$$
S W(\mathcal{L}, H,-1)=-S W(\mathcal{L}, H, 1)
$$

as changing the base orientation changes the orientation of $\operatorname{det}^{+}$. Therefore

$$
S W(\mathcal{L}, H, 0)=0 \quad \text { and } \quad S W(\mathcal{L}, H, 1)=W C N(\mathcal{L}, H) .
$$


We also learn from this proposition that the wall crossing number calculation gives us the invariants themselves instead of their differences. Notice that this discussion does not apply to other types of invariants as other type of invariants may have different parity under the orientation reversion of the base. On the other hand, the argument offered here is very general.

Now we would like to discuss how to transform our question to a question which is closely related to algebraic geometry. For simplicity, let us focus on the $b_{1}=0$ case first.

Let us look at the family index expression that we want to evaluate, it is given by

$$
\int_{\tilde{M}[n] / M[n]} A_{\tilde{M}[n] / M[n]} \cdot \operatorname{ch} \frac{1}{2}(\mathcal{L}) .
$$

Even though we evaluate this as a topological number, we will make use of the fact that $M$ is an almost complex manifold. Writing $\mathcal{L}$ as $K^{-1} \otimes C^{2}$, then (4.7) can be recasted into

$$
\int_{\tilde{M}[n] / M[n]} \operatorname{Todd}_{\tilde{M}[n] / M[n]} \cdot \operatorname{ch}(C) .
$$

And then the Family index theorem becomes Grothendieck RR theorem in the almost complex category. $C$ can be written as $C_{0}-\sum_{i} m_{i} E_{i}$ where $C_{0}$ is a line bundle on $M$ and $E_{i}$ are these line bundles dual to the exceptional divisors.

To evaluate (4.8), we would like to use the Koszul resolution of certain "sheaves" in the almost complex category. For this purpose, let us review the usual Koszul resolution in complex geometry. Let $A \subset B$ be a pair of complex manifolds such that $A$ is imbedded as a complex codimension $d$ submanifold and is the zero set of a section of a locally free sheaf $Q$ of rank $d$. Then the structure sheaf $\mathcal{O}_{A}$ of $A$, extended by zero, is a sky-scraper sheaf on $B$. There is a canonical resolution of $\mathcal{O}_{A}$ in terms of locally free sheaves,

$$
0 \mapsto \Lambda^{d} Q^{*} \mapsto \cdots \Lambda^{2} Q^{*} \mapsto Q^{*} \mapsto \mathcal{O}_{B} \mapsto \mathcal{O}_{A} \mapsto 0
$$

which is usually called the Koszul resolution. As was remarked at the end of the Atiyah-Hirzebruch's paper, $[\mathrm{AH}]$, this resolution goes over to the differentiable category as well. Thus it exists even though the manifold is almost complex. Applying to our special case ( $d$ is equal to one) we get

$$
0 \mapsto \mathcal{O}\left(-E_{i}\right) \mapsto \mathcal{O} \mapsto \mathcal{O}_{E_{i}} \mapsto 0
$$


Tensoring this sequence with a locally free sheaf $\mathcal{N}$ we get

$$
0 \mapsto \mathcal{N}\left(-E_{i}\right) \mapsto \mathcal{N} \mapsto \mathcal{N}_{E_{i}} \mapsto 0 .
$$

Notice that here we are using the $C^{\infty}$ version of the sheaf theory which was sketched in $[\mathrm{AH}]$.

Let us assume $m_{i} \geq 0$ first. In fact, we apply the sequences to different $E_{i}$ in the reversing order. Namely we apply it first to $\mathcal{N}=C_{0}-\sum_{i \leq n-1} m_{i} E_{i}-$ $\left(m_{n}-1\right) E_{n}$. Then we apply it to $\mathcal{N}=C_{0}-\sum_{i \leq n-1} m_{i} E_{i}-\left(m_{n}-2\right) E_{n}$ until the $E_{n}$ is exhausted. Then we do the similar thing to $E_{n-1}$, etc., until all the $E_{i}$ have been eliminated. In the mean time, a sequence of sheaves supporting at those $E_{i}$ will be generated. What matters here is that we have found a new representative of $\mathcal{O}\left(C_{0}-\sum_{i} m_{i} E_{i}\right)$ in the following form

$$
\begin{aligned}
& \mathcal{O}\left(C_{0}-\sum_{i} m_{i} E_{i}\right) \\
& \equiv \mathcal{O}\left(C_{0}\right)-\sum_{i, j_{i} ; 0 \leq j_{i} \leq m_{i}-1} \mathcal{O}_{E_{i}}\left(C_{0}-\sum_{s \leq i-1} m_{s} E_{s}-j_{i} E_{i}\right) .
\end{aligned}
$$

As the Chern character is an additive homomorphism from the $K$ group to the cohomology ring, the family index calculation is reduced to the following form.

$$
\begin{aligned}
\int_{\tilde{M}[n] / M[n]} \operatorname{Todd}_{\tilde{M}[n] / M[n]}\left(\operatorname{ch}\left(C_{0}\right)\right. & \\
& \left.-\sum_{i, j_{i} ; 0 \leq j_{i} \leq m_{i}-1} \mathcal{O}_{E_{i}}\left(C_{0}-\sum_{s \leq i-1} m_{s} E_{s}-j_{i} E_{i}\right)\right) .
\end{aligned}
$$

The first term of (4.9) is intepreted as the index bundle of the $\bar{\partial}$ operator on the fibers $M \sharp n \overline{C P^{2}}$ with the coefficient line bundle $C_{0}$. As $C_{0}$ is pulled back to $\tilde{M}[n]$ by its projection to $M \times M[n]$, the index bundle is a constant bundle on $M[n]$ whose rank is equal to ind $\left(\bar{\partial}, C_{0}\right)$ on $M$. On the other hand, all the contributions of the Chern characters come from the terms which appear with a negative sign. As the sky-scraper sheaves appear in the Chern character, we can restrict our total space from $\tilde{M}[n]$ to $E_{i}$. By the Grothendieck-Riemann-Roch theorem in the differentiable content (see $[\mathrm{AH}]$ for example), the remaining terms can be casted into the following 
form

$$
-\oplus_{i, j_{i} ; 0 \leq j_{i} \leq m_{i}-1} \operatorname{Ind}\left(\bar{\partial},\left(\mathcal{O}\left(j_{i}\right)\right)\right) \otimes\left(C_{0}-\sum_{s \leq i-1} m_{s} E_{s}\right)
$$

where the index is taken over the fibers of $E_{i}$ over $M[n]$ which are unions of normal crossing rational curves.

This formula (4.10) can again be casted into another form. To do so, it requires us introduce some notation. Inductively, let us consider the manifold $M \times M[n]$ with the first $i-1$-th graphs blown up, denoted as $\mathcal{X}_{i-1}$. Then the proper transformations of the $i-$ th graph still forms a section of the projection map from the blown up manifold $\mathcal{X}_{i-1}$ to $M[n]$. Thus we can identify the restriction of the vertical tangent bundle (complex two dimensional) to this section as a complex rank two vector bundle on $M[n]$ which will be denoted by $V_{i}$. Written in terms of $V_{i}$, the formula (4.10) can be expressed as

$$
-\oplus_{i, j_{i} ; 0 \leq j_{i} \leq m_{i}-1} S^{j_{i}}\left(V_{i}^{*}\right) \otimes\left(C_{0}-\sum_{s \leq i-1} m_{s} E_{s}\right) .
$$

where $S^{j_{i}}\left(V_{i}^{*}\right)$ denotes the $j_{i}$-th symmetric power of $V_{i}^{*}$. Thus the wall crossing number should be expressed as $c_{2 n}$ of this vector bundle.

If $m_{i}<0$, then the above discussion is not completely valid. In this case the $\operatorname{Spin}^{c}$ structure can be rewritten as $\mathcal{L}_{0}+\sum_{i}\left(2 m_{i}+1\right) E_{i}$ with $m_{i}$ positive. In this case we still use the same exact sequence

$$
0 \mapsto \mathcal{O}\left(-E_{i}\right) \mapsto \mathcal{O} \mapsto \mathcal{O}_{E_{i}} \mapsto 0
$$

But we replace the middle term by the first and the third term. Thus the term

$$
\sum_{i, j_{i}, 0 \leq j_{i} \leq m_{i}} \mathcal{O}_{E_{i}}\left(C_{0}+\sum_{s \leq i-1}\left(m_{s}+1\right) E_{s}+j_{i} E_{i}\right)
$$

still keeps the form similiar to the original one. The major difference is that it appears with a positive sign instead.

Also $m_{i}$ is replaced by $m_{i}+1$. The key term is written as

$$
\oplus_{i, j_{i}, 0 \leq j_{i} \leq m_{i}} \operatorname{Ind}\left(\bar{\partial},\left(\mathcal{O}\left(-j_{i}\right)\right)\right) \otimes\left(C_{0}+\sum_{s \leq i-1}\left(m_{s}+1\right) E_{s}\right) .
$$


We can go through the previous argument. This time the line bundles restrict to negative degree line bundles on the exceptional $C P^{1}$. Recall that the sheaf cohomology of a negative degree line bundle on $C P^{1}$ is related to the sheaf cohomology of the positive ones by the well known Serre duality on curves. We can formally write

$$
\begin{aligned}
& {\left[H^{0}\left(C P^{1}, \mathcal{O}(-k)\right)\right]-\left[H^{1}\left(C P^{1}, \mathcal{O}(-k)\right)\right]} \\
& =-\left[H^{1}\left(C P^{1}, \mathcal{O}(k-2)\right)\right]^{*}+\left[H^{0}\left(C P^{1}, \mathcal{O}(k-2)\right)\right]^{*} .
\end{aligned}
$$

Apply this formalism to the family of bundles, we still get a series of symmetric powers. The highest power one can get is $m_{i}+1-2=m_{i}-1$, the same index as before. Moreover the Serre duality gives us an extra minus sign. Thus the answer appears with a negative sign, again compatible with the previous answer. Thus we conclude the surprising answer that these line bundles $\mathcal{L}_{0}+\sum_{i}\left(2 m_{i}+1\right) E_{i}, \mathcal{L}_{0}-\sum_{i}\left(2 m_{i}+1\right) E_{i}$ share the same family wall crossing number for the section invariants.

At this moment, let us restrict to the case where $M$ is a complex manifold (including the case when $M$ is the $\mathrm{K} 3$ surface or $T^{4}$ ). Then (4.10) can be interpreted as

$$
-\oplus_{i, j_{i} ; 0 \leq j_{i} \leq m_{i}-1} H^{0}\left(E_{i b},\left(C_{0}-\sum_{s \leq i-1} m_{s} E_{s}\right) \otimes \mathcal{O}_{E_{i b}}\left(j_{i}\right)\right) .
$$

It is this expression which has an interesting algebro-geometric meaning. The first term of the index bundle is nothing else but $H^{0}\left(M, C_{0}\right)$, the space of holomorphic sections of the line bundle $C_{0}$. The term which is substracted actually records the $m_{i}$ jets information of the line bundle $C_{0}$ at the $n$ points of $M$ parametrized by the point in the base $M[n]$. There is a natural map from the tautological line bundle over $P\left(H^{0}\left(M, C_{0}\right)\right)$ to the jet bundle such that this morphism vanishes at a point in $P\left(H^{0}\left(M, C_{0}\right)\right) \times M[n]$ exactly when the corresponding section in $H^{0}\left(M, C_{0}\right)$ vanishes at these $n$ points with prescribed orders. In other words, the counting of such points on the manifold $P\left(H^{0}\left(M, C_{0}\right)\right) \times M[n]$ is equivalent to counting of holomorphic curves (given by the linear system corresponding to $C_{0}$ ) which has certain singularities along the $n$ points. Thus we have seen in these type of examples that the algebro-geometric problem is actually encoded in a differential topological invariant. This gives us a very nontrivial example that the family version of Seiberg-Witten theory should have certain Gromov type interpretation. 
Let us come back to the almost complex category. In the previous discussion, we have made the assumption $b_{1}=0$ to simplify the discussion. Now let us consider the general $b_{1} \neq 0$ case.

When $b_{1}=0$, the torus $T^{b_{1}}$ collapses to a point and it does not play any role in the formula. When $b_{1} \neq 0$, the key observation is that the torus fibration is reduced to $T^{b_{1}} \times \tilde{M}[n]$ in this special situation. Even though the fiber bundle $\tilde{M}[n] \mapsto M[n]$ is not a trivial product bundle, the nontriviality is due to the blow up process which has no effect on the $H^{1}$, nor on the flat connections on the manifold $M$. Therefore the torus fibration should be the same as the one for $M \times M[n] \mapsto M[n]$, the trivial product.

The factorization of the torus fibration leads to certain simplifications of the calculations. Previously, the Index virtual bundle was splitted into two parts. The positive part is a trivial bundle over the base and the negative part carries all the topological information. In the $b_{1}>0$ case the same conclusion is not valid. As the twisting factor $\operatorname{ch}(\Omega)$ play sa role, the positive factor is replaced by a virtual bundle of constant rank. This virtual bundle can be written as the formal difference of two terms. The negative part is still a trivial bundle. However the positive part is not. If we take a closer look at the bundle involved, it is exactly the bundle which appears in the family wall crossing formula of $\mathcal{L}_{0}$ in the fiber bundle $M \times p t\left(b^{+}=1\right)$ or $M \times S^{b^{+}-1}\left(b^{+}>1\right)$. This vector bundle has a special feature that it is trivial along the factor $M[n]$. On the other hand the negative part of the index bundle is still given by the original expression discussed above. It also has a special feature that it is trivial along the $T^{b_{1}}$ factor. Thus we get a nice picture out of the complicated situation. Namely we are working on a product space $T^{b_{1}} \times M[n]$ and the index bundle is decomposed into terms which are pulled back from $T^{b_{1}}$ and $M[n]$ respectively.

Let us denote the projection map from $T^{b_{1}} \times M[n]$ to $T^{b_{1}}$ and $M[n]$ by $\pi_{T}$ and $\pi_{M}$ respectively. Then the index bundle can be written as

$$
I N D_{M[n]}(D)=\pi_{T}^{*} W_{1}-\pi_{M}^{*} W_{2}
$$

where $W_{1} \in K\left(T^{b_{1}}\right)$ and $W_{2} \in K(M[n])$.

To calculate the Segre classes of the index bundle $S\left(I N D_{M[n]}(D)\right)$, we would like to make use of the product structure described here. Instead of doing it directly, we can rewrite (4.13) as

$$
I N D_{M[n]}(D) \oplus \pi_{M}^{*} W_{2}=\pi_{T}^{*} W_{1} .
$$

As Segre classes are the formal inverse of the Chern classes, they also satisfy the Whitney formula. Thus we have $S\left(I N D_{M[n]}(D)\right) \cdot S\left(\pi_{M}^{*} W_{2}\right)=$ 
$S\left(\pi_{T}^{*} W_{1}\right)$. Multiplying both sides by $C\left(\pi_{M}^{*} W_{2}\right)$ and make use of the $S \cdot C=1$ property we finally get

$$
S\left(I N D_{M[n]}(D)\right)=S\left(\pi_{T}^{*} W_{1}\right) \cdot C\left(\pi_{M}^{*} W_{2}\right) .
$$

If we are interested in the general terms of the left hand side, it is expressed in terms of the combinations of the Chern classes and Segre classes of $W_{1}$ and $W_{2}$ in a cubermsome way. However the result we are interested at is the top Segre class (where the word "top" means the top dimension of $\left.T^{b_{1}} \times M[n]\right)$. In this case the formula simplifies and it is given by the top Segre class of $W_{1}$ and the top chern class of $W_{2}$. We also notice that the top Segre class of $W_{1}$ is nothing else but the wall crossing number of $\mathcal{L}_{0}$ in the critical product family. Thus, we have gotten an amazing result relating the family wall crossing formula of the fiber bundle $\tilde{M}[n]\left(\right.$ or $\tilde{M}[n] \times S^{b^{+}-1}$ ) and the family wall crossing formula of $M$ or $M \times S^{b^{+}-1}$. The following is the main theorem of this subsection.

Theorem 4.16. Let $\delta_{M[n]}(\mathcal{L})$ be the wall crossing number of the consecutive chambers of the family $\tilde{M}[n]$ or $\tilde{M}[n] \times S^{b^{+}-1}$. Let $\mathcal{L}$ be written as $\mathcal{L}_{0}+$ $\sum_{i}\left(2 m_{i}+1\right) E_{i}$ with $m_{i} \geq 0$, then $\delta_{M[n]}(\mathcal{L})$ can be written as the following expression in terms of $\delta\left(\mathcal{L}_{0}\right)$, the wall crossing number of the consecutive chamber of the family $M \times p t$ or $M \times S^{b^{+}-1}$,

$$
\begin{aligned}
& \delta_{M[n]}(\mathcal{L}) \\
& =\int_{M[n]} c_{2 n}\left(\oplus_{i, j_{i} ; 0 \leq j_{i} \leq m_{i}-1} S^{j_{i}}\left(V_{i}^{*}\right) \otimes\left(C_{0}-\sum^{s \leq i-1} m_{s} E_{s}\right)\right) \cdot \delta\left(\mathcal{L}_{0}\right),
\end{aligned}
$$

where $\mathcal{L}_{0}=2 C_{0}-K_{M}$. If $2 m_{i}+1$ is negative in the formula of $\mathcal{L}$, then its wall crossing number is the same as the one with $-\left(2 m_{i}+1\right)$ replacing $2 m_{i}+1$. Namely there is an explicit $\mathbf{Z}_{2}$ symmetry among the Spinc structures which preserves the wall crossing number.

When $b_{1}(M)=0$, the wall crossing number of the critical family becomes \pm 1 and the formula goes back to the expression studied earlier. We want to emphasize that only in the $b_{1} \neq 0$ case does the dependence on $\delta\left(\mathcal{L}_{0}\right)$ become explicit.

The theorem is not only valid for the pure family Seiberg Witten invariant, it is valid for the invariant involving $H_{1}(M ; \mathbf{Z})$ as well, as long as $\delta\left(\mathcal{L}_{0}\right)$ 
is understood to be the wall crossing number of the corresponding invariant of the critical family. $\delta\left(\mathcal{L}_{0}\right)$

On the other hand, if we are interested in the wall crossing numbers of the mixed invariants, it is easy to derive the corresponding wall crossing formula. Let $\eta$ be the cohomology class in $H^{*}(M[n] ; \mathbf{Z})$ whose insertion defines the mixed Seiberg-Witten invariants. Then the wall crossing formula is of almost the same form except that $\int_{M[n]} c_{2 n}(\cdots)$ is replaced by $\int_{M[n]} \eta$. $c_{2 n-\operatorname{deg} \eta}(\cdots)$. Especially the wall crossing formula for the mixed invariant with the fundamental class $[M[n]] \in H^{\text {top }}(M[n] ; \mathbf{Z})$ inserted is the same as the wall crossing formula $\delta\left(\mathcal{L}_{0}\right)$.

There are several things we learn from the derivation of the wall crossing formula for these special families constructed out of the Fulton-MacPherson spaces. First, the lengthy formula in terms of all the Chern characters may not be so useful in deriving the formula. One may need to figure out certain tricks (in our case, the Koszul resolution of sheaves in the smooth category) to simplify the calculation. As the final answer depends on the topology of the fiber bundle, one should not expect the answer to be as universal as in the critical case. To calculate the formula for the other interesting family is an interesting question of its own.

Second, as the wall crossing formula satisfies the amazing factorization property, one may suspect that the Seiberg-Witten invariants themselves also satisfy the similiar constraints. Namely they are related to the family invariants of the critical families in an explicit way. In fact, the family blow up formula [Liu] supports this. Using the family blow up formula, one is able to check that the family Seiberg-Witten invariants satisfy the constraint predicted here. For the manifolds $M$ with $b^{+}-1$ positive and even, the result could be gotten by the wall crossing formula and the vanishing result stated earlier. However the family blow up formula gives us an independent derivation valid for all cases (including the $b^{+}=1$ case). The compatibility of the blow up formula and the wall crossing formula gives us a commutative diagram between blowing up and crossing chambers. Let us begin from the fiber bundle $M \times M[n] \times S^{b^{+}-1}$. One can either move from the zero winding chamber to the nonzero winding chamber and then blow up to $\tilde{M}[n] \times S^{b^{+}-1}$. Or one can first blow up $n$ times to $\tilde{M}[n] \times S^{b^{+}-1}$ and then go across the walls to the nonzero winding chambers. The family blow up formula also explains why the vanishing result holds for the zero winding chamber. Once the pure invariants of the zero winding chamber of the critical families are known to be zero, the others follow as well by the family blow up formula. For the details please consult [Liu]. 
Finally, let us give the explicit formulas when $n=1$. When $n=1, M[n]$ is simply $M$, and $V_{1}$ is just the tangent bundle. Consider the case $m_{1}=2$, then by (4.14)

$$
\begin{aligned}
\delta_{M}(\mathcal{L}) & =\delta\left(\mathcal{L}_{0}\right) \int_{M} c_{2}\left(\left(S^{0}(T M) \oplus S^{1}(T M)\right) \otimes C_{0}\right) \\
& =\delta\left(\mathcal{L}_{0}\right)\left(3 C_{0} \cdot C_{0}+2 C_{0} \cdot c_{1}(M)+c_{2}(M)\right)
\end{aligned}
$$

When $M$ is the four torus, both $c_{1}(M)$ and $c_{2}(M)$ are trivial, so by Corollary 4.11,

$$
\begin{aligned}
\delta_{M}(\mathcal{L}) & =\delta\left(\mathcal{L}_{0}\right) 3 C_{0} \cdot C_{0} \\
& =\frac{1}{2}\left(C_{0} \cdot C_{0}+1\right) 3 C_{0} \cdot C_{0} .
\end{aligned}
$$

In [BL2], for a hyperkahler family of four torus, the number of 1-nodal curves in a primitive class $C_{0}$, is exactly given by (4.15). This is again an evidence supporting 'SW=TGW'.

\section{References.}

[AH] M. Atiyah and F. Hirzebruch, The Riemann-Roch theorem for analytic embeddings, Topology, 1 (1962) 151-166.

[BL1] J. Bryan and C. Leung, The enumerative geometry of K3 surfaces and modular forms, preprint.

[BL2] J. Bryan and C. Leung, Generating functions for the number of curves on Abelian surfaces, preprint.

[D1] S. Donaldson, The Yang-Mills invariants of four-manifolds, in 'Geometry of low-dimensional manifolds, I (Durham, 1989)', 5-40, London Math. Soc. Lecture Note Ser., 150, Cambridge Univ. Press, Cambridge, 1990.

[D2] S. Donaldson, The Seiberg-Witten equations and 4-manifold topology, Bull. AMS, 33(1) (1996), 45-70.

[DK] S. Donaldson and P. Kronheimer, The geometry of four-manifolds, Oxford Mathematical Monographs, Oxford Science Publications, The Clarendon Press, Oxford University Press, New York, 1990.

[FS] R. Fintushel and R. Stern, Knots, links, and 4-manifolds, preprint. 
[F] W. Fulton, Intersection theory. Ergebnisse der Mathematik und ihrer Grenzgebiete (3), [Results in Mathematics and Related Areas (3)], 2, Springer-Verlag, Berlin, 1984.

[FM] W. Fulton and R. MacPherson, A compactification of configuration spaces, Ann. of Math., 139 (1994), 183-225.

[FMo] R. Friedman and J. Morgan, Algebraic surfaces and Seiberg-Witten invariants, preprint.

[Ge] H. Geiges, Symplectic couples on 4-manfolds, Duke. Math. Journal, 85 (1996), 701-711.

[Gr] M. Gromov, Pseudo-holomorphic curves in symplectic manifolds, Invent. Math., 82 (1985), 307-347.

[GS] V. Guillemin and S. Sternberg, Birational equivalence in the symplectic category, Invent. Math., 1989.

[IP] E-N. Ionel and T. Parker, The Gromov invariants of Ruan-Tian and Taubes, preprint.

[K] P. Kronheimer, Some nontrivial families of symplectic structures, preprint.

[KM] P. Kronheimer and T. Mrowka, The genus of embedded surfaces in the projective plane, Math. Res. Letters, 1 (1994), 797-808.

[Liu] A. Liu, Family blow up formula and nodal curves in Kahler surfaces, preprint.

[LL1] T.J. Li and A. Liu, General wall crossing formula, Math. Res. Letter, 2 (1995).

[LL2] T.J. Li and A. Liu, On the equivalence between $S W$ and $G T$ in the case $b^{+}=1$, to appear in IMRN.

[LL3] T.J. Li and A. Liu, Symplectic four-manifolds with torsion canonical classes, in preparation.

[M] D. McDuff, Lectures on Gromov invariants for symplectic 4-manifolds, to appear in the Proc. NATO Summer School, Montreal.

[M2] D. McDuff, The structure of rational and ruled symplectic 4-manifold, J. AMS, 1(3) (1990), 679-710. 
[R] Y. Ruan, Virtual neighborhoods and pseudo-holomorphic curves, in 'Proceedings of 6th Gökova Geometry-Topology Conference', Turkish J. Math., 23(1) (1999), 161-231.

[RT] Y. Ruan and G. Tian, A mathematical theory of quantum cohomology, J. Differential Geom., 42(2) (1995), 259-367.

[Rub] D. Ruberman, An oberstuction to smooth isotopy in dimension 4, preprint.

[S] N. Steenrod, Topology of fibre bundles, Princeton University Press, Priceton, 1951.

[SW] N. Seiberg and E. Witten, Electric-magnetic duality, monople condesation, and confinement in $N=2$ supersymmetric Yang-Mills theory, Nuclear Physics, B426 (1994), 19-52.

[T1] C.H. Taubes, The Seiberg-Witten invariants and symplectic forms, Math. Res. Letters, 1 (1994), 809-822.

[T2] C.H. Taubes, SW $\Rightarrow$ Gr: From the Seiberg-Witten equations to pseudoholomorphic curves, Jour. Amer. Math. Soc., 9 (1996), 845-918.

[T3] C.H. Taubes, Counting pseudo-holomorphic submanifolds in dimension four, J. Diff. Geom., 44(4) (1996), 818-893.

[T4] C.H. Taubes, Gr $\Rightarrow \mathrm{SW}$ : From Pseudo-holomorphic curves to the Seiberg-Witten invariants, preprint.

[T5] C.H. Taubes, Gr=SW, Counting curves and connections, preprint.

[W] E. Witten, Monoples and four-manifolds, Math. Res. Lett., 1 (1994), 769-796.

[YZ] S.T. Yau and E. Zaslow, BPS states, string duality, and nodal curves on K3, Nuc. Phys., B471(3) (1996), 503-512.

Math. Department

Princeton University, Princeton, NJ 08544

E-mail address: tli@math.princeton.edu

Math. Department

UC Berkeley, Berkeley, CA 94720

E-mail address: akliu@math.berkeley.edu

ReCeived April 13, 1999. 